\title{
Volitional modulation of higher-order visual cortex alters human perception
}

Article

Accepted Version

Ekanayake, J., Ridgway, G. R., Winston, J. S., Feredoes, E., Razi, A., Koush, Y., Scharnowski, F., Weiskopf, N. and Rees, G. (2019) Volitional modulation of higher-order visual cortex alters human perception. Neurolmage, 188. pp. 291-301. ISSN 1053-8119 doi:

https://doi.org/10.1016/j.neuroimage.2018.11.054 Available at https://centaur.reading.ac.uk/81314/

It is advisable to refer to the publisher's version if you intend to cite from the work. See Guidance on citing.

To link to this article DOI: http://dx.doi.org/10.1016/j.neuroimage.2018.11.054

Publisher: Elsevier

All outputs in CentAUR are protected by Intellectual Property Rights law, including copyright law. Copyright and IPR is retained by the creators or other copyright holders. Terms and conditions for use of this material are defined in the End User Agreement.

www.reading.ac.uk/centaur

\section{CentAUR}


Central Archive at the University of Reading

Reading's research outputs online 
1 TITLE:

2

3 Volitional Modulation of Higher-order Visual Cortex Alters Human Perception

4

5 AUTHORS:

6

7 Jinendra Ekanayake ${ }^{1,2}$, Gerard R Ridgway ${ }^{3}$, Joel $S$ Winston ${ }^{1,2}$, Eva

8 Feredoes $^{4}$, Adeel Razi ${ }^{1,9}$, Yury Koush ${ }^{10}$, Frank Scharnowski ${ }^{5,6,7}$, Nikolaus

9 Weiskopf $^{1,8}$, Geraint Rees ${ }^{1,2}$

10

11 AFFILIATIONS:

12

13 'Wellcome Trust Centre for Human Neuroimaging, UCL Institute of Neurology,

14 London, UK

$15{ }^{2}$ UCL Institute of Cognitive Neuroscience, University College London

$16{ }^{3}$ Oxford Centre for Functional MRI of the Brain, University of Oxford, Oxford, UK

$17 \quad{ }^{4}$ School of Psychology and Language Sciences, University of Reading, UK

$18{ }^{5}$ Psychiatric University Hospital, University of Zürich, Lenggstrasse 31, 8032 Zürich,

19 Switzerland

$20{ }^{6}$ Neuroscience Center Zürich, University of Zürich and Swiss Federal Institute of

21 Technology, Winterthurerstr. 190, 8057 Zürich, Switzerland

22 'Zürich Center for Integrative Human Physiology (ZIHP), University of Zürich,

23 Winterthurerstr. 190, 8057 Zürich, Switzerland

$24{ }^{8}$ Department of Neurophysics, Max Planck Institute for Human Cognitive and Brain

25 Sciences, Leipzig, Germany

$26{ }^{9}$ Department of Electronic Engineering, NED University of Engineering and 27 Technology, Karachi, Pakistan 
$28{ }^{10}$ Department of Radiology and Biomedical Imaging, Yale University, 300 Cedar

29 Street, New Haven, CT 06519, USA

30

31 CORRESPONDING AUTHOR:

32 Jinendra Ekanayake, email: j.ekanayake@ucl.ac.uk

33 ADDRESS:

34 Institute of Cognitive Neuroscience,

3512 Queen Square, WC1N 3AR

36

37 Conflict of Interest: Nil

38 


\section{Abstract}

41 Can we change our perception by controlling our brain activation? Awareness

42 during binocular rivalry is shaped by the alternating perception of different

43 stimuli presented separately to each monocular view. We tested the possibility

44 of causally influencing the likelihood of a stimulus entering awareness. To do

45 this, participants were trained with neurofeedback, using realtime functional

46 magnetic resonance imaging ( $r t-f M R I)$, to differentially modulate activation in

47 stimulus-selective visual cortex representing each of the monocular images.

48 Neurofeedback training led to altered bistable perception associated with 49 activity changes in the trained regions. The degree to which training

50 influenced perception predicted changes in grey and white matter volumes of

51 these regions. Short-term intensive neurofeedback training therefore sculpted

52 the dynamics of visual awareness, with associated plasticity in the human 53 brain.

54

55 Word count $123(150$ max $)$

56

57

58 
61 - Unconscious biasing of higher-order visual perception with realtime $62 \quad \mathrm{fMRI}$ neurofeedback.

63 - Participants unknowingly modulated two brain regions to control 64 feedback signal

65 - Short-term neurofeedback training over 3 days induced functional $66 \quad$ plasticity

67 - Neurofeedback may strengthen neural representations and alter prior $68 \quad$ expectations

69 - Potential avenue for behavioural shaping and therapeutic reduction of 70 aberrant perception

71

72 
75 The ability to causally modify how we perceive the world has potential

76 implications in health and disease. Altering perceptual biases, which may be

77 conscious or unconscious, could modify pathological perception such as

78 hallucinations, or provide a means of selective cognitive

79 enhancement(Miranda et al., 2015). Such attempts to deliberately manipulate

80 higher-order sensory perception have, until now, proven to be unsuccessful.

81 For example, attempting to alter perception using mental imagery, a cognitive

82 process which utilises similar neural substrates to perception(O'Craven and 83 Kanwisher, 2000), does not increase the vividness of the imagery. Most 84 importantly, mental imagery training has no effect on perception linked to the 85 imagery strategy used during training, as demonstrated with binocular rivalry 86 (BR) between images specifically associated with the mental imagery 87 training(Rademaker and Pearson, 2012). BR is a unique perceptual 88 phenomenon that has been used to provide a window into the unconscious 89 and conscious processes underlying visual perception. It is produced by 90 simultaneously presenting conflicting monocular stimuli to each eye.

91 Paradoxically, the brain cannot form a stable image. Instead, each image

92 randomly competes for exclusive perceptual dominance. Until now, producing 93 unconscious shifts in higher-order perception by directly modifying brain 94 function has proven to be unsuccessful.

95

96 Neurofeedback training using realtime functional magnetic resonance imaging 97 (rt-fMRI) is an emerging technique which allows participants to control target 
98 brain regions by voluntarily modulating online feedback of activity in those 99 regions(Sitaram et al., 2016). Feedback is typically provided via a visual 100 interface during concurrent MR scanning. Online modulation of the Blood 101 Oxygen Level-Dependent (BOLD) signal using neurofeedback involves 102 abstract cognitive strategies, as well as mental imagery that maybe explicitly 103 linked to the brain region-of-interest (ROI). This approach can produce 104 changes in behaviour through the functional modulation of trained brain 105 regions, including low-order visual perception (e.g. grating orientation, colour) 106 by modulating primary retinotopic cortex(Amano et al., 2016; Shibata et al., 107 2011), pain and craving by modulating anterior cingulate cortex(deCharms et 108 al., 2004; Li et al., 2013), and motor function by modulating supplementary 109 motor area and primary motor cortex(Blefari et al., 2015; Subramanian et al., 110 2011). We hypothesised that rt-fMRI neurofeedback might prove more 111 powerful than previous approaches, such as mental imagery alone, in 112 enabling participants to modify brain activity associated with higher-order 113 visual perception, and consequently directly influence how they perceive the 114 world.

116 To test this hypothesis, we trained human participants using mental imagery 117 combined with neurofeedback to voluntarily control the difference in activation 118 between two higher-order visual cortical regions (Fusiform Face Area, FFA 119 and Parahippocampal Place area, PPA). The human FFA responds strongly 120 to faces(Kanwisher et al., 1997; McCarthy et al., 1997), but not to other types 121 of non-face stimuli, while the PPA responds to houses and places, but not 122 faces(Epstein and Kanwisher, 1998). Further, both of these regions activate 
123 during mental imagery of faces or places respectively, even in the absence of 124 visual stimuli(O'Craven and Kanwisher, 2000). The differential response 125 properties of these two regions enabled participants in the study to have a 126 visually presented neurofeedback training signal that represented the 127 difference in activation between the two regions i.e. a differential signal.

129 The use of a differential signal provided an internal control for global brain 130 activation, and helped focus the training effect on the two selected brain 131 regions in a manner that might not occur with mental imagery training only. 132 We tested participants with a BR task, where they were exposed to rivalrous 133 monocular face and house images, before and after neurofeedback training. 134 During BR, participants are consciously aware of only one of the perceptual 135 stimuli at a time, while the other stimulus is temporarily suppressed. The 136 perceptual fluctuation is spontaneous and stochastic, with both top-down (i.e. 137 cognitive modulation) and bottom-up (i.e. salience-based) processes being 138 implicated(Dayan, 1998; Parker and Alais, 2007; Tong et al., 2006). In this 139 study, the ensuing BR, where perception alternated spontaneously between 140 each monocular view, provided a test of whether neurofeedback training had 141 altered the likelihood of either stimuli entering awareness. We investigated 142 whether any perceptual changes were associated with differences in brain 143 activity and structure (see also Supplementary Materials).

145 To anticipate our findings, following neurofeedback training, there was a 146 sustained influence on the perceptual dynamics of BR, suggesting functional 147 plasticity. This effect was additionally observed when participants performed 
148 concurrent modulation of brain activity during BR. Further, a multivariate 149 analysis of changes in brain structure produced by neurofeedback training 150 predicted changes in BR dynamics. 
177 stimuli were displayed side-by-side on the monitor, each with a central white

178 fixation cross (0.68 visual angle) and tile frame surround (11.78 visual angle),

\section{Main experiment:}

\section{Participants}

Ten neurologically normal adult volunteers (24-35 years of age; mean age 28 years; 8 females) with normal or corrected-to-normal visual acuity participated in the experiment. Each participant gave written informed consent. The study was approved by the local ethics committee (UCL Ethics Committee code: 09/H0716/14).

\section{Stimuli and Materials}

All visual stimuli were generated and displayed via scripts in MATLAB created with the Cogent 2000 toolbox (http://www.vislab.ucl.ac.uk/cogent_2000.php), on a viewing screen with a visual angle of 23 degrees by 17 degrees, $(30 \times 26$ LCD projector (LT158; NEC). The mirror-mounted viewing screen was set on the top of the scanner bore (optical distance $52 \mathrm{~cm}$ ). During the neurofeedback sessions, participants saw a fluctuating thermometer bar at the centre of the screen. During the BR sessions only, participants wore a pair of prism glasses. Additionally, a black cardboard divider was placed between the forehead and the screen to ensure that each eye could see one side of the screen only, and provide a stable base for fixation. Two identical box

fixation cross (0.68 visual angle) and tile frame suround (11.78 visual angle), 
upon a uniform grey background (background luminance $1 / 465 \quad 6 d / \mathrm{m}^{2}$ ).

180 Optimal perceptual fusion of the two box stimulus images was confirmed with

181 the participant prior to commencing each BR session. Face or house stimuli 182 were presented (20 exemplars each). Responses for durations were obtained 183 via a pair of custom-built, MR-compatible, response boxes.

\section{FMRI Scanning}

Scanning was performed on a 3T Allegra head-only scanner (standard 188 transmit-receive head coil). Functional data was acquired with a single-shot 189 gradient echo planar imaging sequence (matrix size, 64x64; field of view, $190192 \times 192 \mathrm{~mm}$; isotropic in-plane resolution, 3x3 mm; 32 slices with ascending 191 acquisition; slice thickness, $2 \mathrm{~mm}$; slice gap, $1 \mathrm{~mm}$; echo time (TE), $30 \mathrm{~ms}$; 192 repetition time (TR), $1920 \mathrm{~ms}$; flip angle, 90; receiver bandwidth, 3551 $193 \mathrm{~Hz} /$ pixel). Although the nominal slice thickness was $2 \mathrm{~mm}$, the effective slice 194 profile achieved in practice is typically larger such that the effective slice 195 thickness is closer to $3 \mathrm{~mm}$. Allowing a gap additionally minimised any risk of 196 saturation effects upon excitation of the subsequent slice (again due to 197 imperfect slice profiles). This is particularly important in the case of ascending 198 acquisition order, as used here. Ascending acquisition order was chosen to 199 minimise the impact of any participant motion, which again could lead to 200 saturation effects if the motion resulted in any part of the previously excited 201 slice being re-excited in a time shorter than the TR.

202

203 Within each scanning session, double-echo fast, low-angle shot sequence 
204 (FLASH) field maps (TE1, $10 \mathrm{~ms}$; TE2, $12.46 \mathrm{~ms}$; resolution, $3 \times 3 \times 2$ mm;

205 slice gap, $1 \mathrm{~mm}$ ) were acquired and used to correct geometric distortions.

206

207 High Resolution Structural Scans

208

209 A whole brain high-resolution T1-weighted structural scan was performed 210 before and after training. This was in addition to structural scans performed on

211 each neurofeedback training day. The scan was a 3D-modified, driven 212 equilibrium Fourier transform (MDEFT) scan (1 $\mathrm{mm}$ isotropic resolution; matrix 213 size, 256x240 mm; field of view, $256 \times 240 \mathrm{~mm}$; 176 sagittal partitions; TE, 2.4 $214 \mathrm{~ms}$; TR, $7.92 \mathrm{~ms}$; inversion time, $910 \mathrm{~ms}$; flip angle, 15; readout bandwidth, $215195 \mathrm{~Hz} /$ pixel; spin tagging in the neck with flip angle $160^{\circ}$ to avoid flow 216 artifacts for superposition of functional maps(Deichmann et al., 2004)).

218 Realtime fMRI Set-up for Neurofeedback

219

220 Turbo Brain Voyager(Goebel et al., 2006) was used, with custom realtime 221 image export tools programmed in ICE VA25 (Siemens Healthcare)(Weiskopf 222 et al., 2004), and custom MATLAB based scripts. Participants were shown 223 visual representations of BOLD signal changes in brain regions previously 224 identified with a functional localiser scan (i.e. target ROls). Realtime data 225 preprocessing encompassed 3D motion correction, smoothing, and 226 incremental linear detrending of time series. The ROI time course(s) were 227 extracted from the prescribed ROI masks, averaged and exported. Signal 228 drift, spikes and high frequency noise were further removed in realtime from 
229 the exported time courses with custom MATLAB scripts(Koush et al., 2012).

230 The feedback signal (a 'fluctuating' thermometer bar) was displayed to the

231 participants with a delay of $2 \mathrm{~s}$ from the acquisition of the image.

232

233 Binocular Rivalry Set-up and Behavioural data acquisition

234

235 Inside the scanner, participants, wearing custom-made prism glasses, were

236 shown two stimuli equidistant from a central viewing screen divider. During

237 the viewing blocks, a face stimulus and a house stimulus were presented in

238 the left and right hemi-fields respectively. The stimuli were pseudorandomised

239 with regards to which eye received the face or house stimuli. Each viewing

240 block (40 s followed by rest $20 \mathrm{~s}$ ) was performed with a new pair of stimuli

241 from the pool of 20 stimuli. Six blocks were performed per session, for three

242 sessions.

243

244 During the BR sessions, participants pressed one of three buttons to record 245 their percept of 'face', 'house' or 'mixed'. The participants were instructed to 246 switch as accurately and rapidly as possible between the three possible 247 button presses linked to the three percepts. This was the only instruction 248 given during pre-training BR and post-training BR, which were identical save 249 for being performed either side of neurofeedback training. Additional 250 instructions were given for two further post-training BR conditions (see below, 251

252 Day 5: Post-training BR).

253 
254 Cumulative dominance durations were calculated, which were equal to the 255 total amount of time each monocular stimulus was perceived, and averaged 256 across blocks. The three percepts were then pooled as follows: (1) strategy257 related percept e.g. face percept for the neurofeedback group advised to use 258 face mental imagery ('Face' group) or house percept for the neurofeedback 259 group advised to use house mental imagery ('House' group) (2) strategy260 unrelated percept' e.g. house percept for the 'Face' group, face percept for 261 the 'House' group); and (3) 'mixed percept'.

\section{Experimental Outline}

265 The experiment was divided into multiple days, with each participant attending 266 five consecutive scanning days (Figure 1). The participants were split into two 267 groups, with five participants in the 'face' group and five participants in the 268 'house' training group.

\section{Day 1: Pre-training BR and Localiser}

271 A Pre-training BR scan was performed as described above for all 272 participants. They then underwent a functional localiser scan to identify FFA 273 and PPA regions (12 minutes, 16 blocks of face stimuli, 16 blocks of house 274 stimuli, and 20 different exemplars per block). Each stimulus was presented 275 for $600 \mathrm{~ms}$ (400 ms interstimulus interval). A one-back task was performed (3 276 targets per block), requiring a button press upon detection of the same 277 stimulus. Two contrasts were used; Houses vs. Faces and Faces vs. Houses. 278 Using the Juelich histological atlas to provide an anatomical 
279 landmarks(Eickhoff et al., 2006, 2005), voxel selection for the ROls were 280 defined along the ventral and lateral surfaces of the temporal lobe in proximity

281 to the fusiform gyrus for FFA, and lateral to the collateral sulcus in the 282 parahippocampal region for PPA respectively.

283

284 Day 2-4: Neurofeedback Sessions

285 Each neurofeedback training day comprised three scanning sessions, each 286 six blocks of $60 \mathrm{~s}$ with an 'upregulate' period (40 s) followed by 'rest' (20 s). 287 During an upregulation period, participants viewed a fluctuating red bar and a 288 fixed horizontal black bar. The latter was placed towards the top of the screen, 289 and the participants were asked to push the red bar above it. Participants 290 were told that the fluctuating red bar was linked to their brain activity, and that 291 they should drive the red bar up to the level of the black bar using a mental 292 imagery strategy. They were advised to maintain the red bar at that level, for 293 as long as possible, during the 'upregulate' period. Participants were told that 294 there was a delay related to the training signal (produced by the 295 hemodynamic response function, HRF) of approximately 6-8 s. During rest, 296 participants were instructed to perform a mental arithmetic task (serial 297 subtraction of 7 from 100).

299 Controlling the Neurofeedback Training Signal

301 Participants were pseudorandomised into two groups - a 'Face' group and a 302 'House' group. Each group was instructed to use mental imagery strategies. 303 They were given examples of what might work (Figure 1), although the 
participants could use their own interpretation. Specific examples for the

305 house group were 'think about your house, or a building you are familiar with

306 such as a school or church', or 'think about walking down the road looking at

307 buildings'. Specific examples for the face group were 'think of faces of people

308 you know', 'think of celebrity faces', or 'think of memorable faces you have

309 seen recently'. Both groups were instructed to pay close attention to the

310 fluctuating red bar, and to find the best way of pushing the bar up for as much

311 and as long as possible. Both groups were instructed to use whatever

312 strategy worked best, including their own, and to vary the strategy to ensure

313 continuous control of the fluctuating red bar.

315 Each group was unaware of the precise nature of their feedback signal.

316 During neurofeedback training, the fluctuating red bar was driven by brain

317 activity in which the signal from PPA was subtracted from FFA for the 'Face'

318 group, and the reverse subtraction (PPA - FFA) for the 'House' group.

319 Participants were trained to modulate a differential training signal. Therefore,

320 the 'Face group' learned to voluntarily increase the difference in BOLD

321 between FFA and PPA. In contrast, the 'House group' learned to voluntarily

322 increase the difference in BOLD between PPA and FFA.

323

324 For each group there was a strategy-related ROI (e.g. FFA for the Face group

325 and a strategy-unrelated ROI (e.g. PPA for the Face group, and vice versa for 326 the House group, Figure 2A).

328 Day 5: Transfer Session 
329 After the final neurofeedback training session, there were two transfer 330 sessions, each comprising six blocks. Each block lasted $60 \mathrm{~s}$ and consisted of

331 an 'upregulate' period (40 s) followed by 'rest' (20 s). During upregulation, 332 participants were required to drive their brain activity 'up', using the mental 333 imagery strategies successfully used to drive the bar during neurofeedback 334 training, but now in the absence of a feedback signal.

335

336 Day 5: Post-training BR

337 All participants then performed post-training BR, with the same set-up 338 described for pre-training BR. Three different BR conditions were performed 339 (2 sessions each) pseudorandomised and counterbalanced across all 340 participants: (1) Post-training BR. The instruction was identical to the pre341 training BR; (2) Post-training BR with 'concurrent trained upregulation'. 342 Both groups were instructed to use their trained mental imagery strategies 343 that had worked best during the training sessions while simultaneously 344 performing BR; and (3) Post-training BR with 'concurrent non-trained 345 mental imagery'. Participants were instructed to use mental imagery related 346 to either houses if in the 'Face group', or faces if in the 'House group'. Mental 347 imagery was to be performed while concurrently performing BR.

\section{Brain Imaging}

351 Functional data was analysed using SPM12 (http://www.fil.ion.ucl.ac.uk/spm). 352 To allow for T1 equilibration the first five images of each session were 353 discarded. Preprocessing involved bias correction, realignment of each EPI to 354 the mean EPI, unwarping, and co-registration of the functional data to the 
355 structural image. Normalisation was not performed, as initial analyses were 356 performed in native space. Data was smoothed with a $6 \mathrm{~mm}$ FWHM Gaussian 357 kernel and high-pass filtered (128s cut-off) to remove low-frequency noise, 358 while at the same time preserving as many of the spontaneous fMRI 359 fluctuations as possible(Cordes et al., 2001). Session-specific grand mean 360 scaling was applied with no global normalisation.

Offline ROI Analysis: Fusiform Face Area and Parahippocampal Place Area

\section{Neurofeedback}

366 BOLD signals across the 9 training sessions (acquired on Days 2-4) were 367 modeled using a GLM, with regressors for each of the 9 sessions. Boxcar 368 functions were created for the six upregulation blocks, convolved with the 369 canonical HRF. Six regressors for movement and a global constant were 370 included. Beta values from the GLM were averaged across all the voxels in 371 the ROI masks (FFA and PPA ROls based on the functional localiser). Mean 372 percentage signal change (PSC) was then calculated. For each participant, 373 the differential mean PSC between the two ROls (i.e. strategy-related ROI 374 minus strategy-unrelated ROI) was calculated across sessions. From this, the 375 average mean PSC across participants over the training was calculated.

\section{Transfer Sessions}

378 Two transfer sessions were performed, with participants performing six blocks 379 of upregulation of brain activity as trained, but now in the absence of a 
neurofeedback signal. In a similar manner to the neurofeedback sessions

381 (see above), the differential mean PSC between the two ROls (i.e. strategy382 related $\mathrm{ROI}$ minus strategy-unrelated $\mathrm{ROI}$ ) was calculated across sessions, 383 and from this, the average mean PSC across participants over transfer was 384 calculated.

385

\section{Binocular Rivalry}

387 Boxcar functions were created to model the onset of the BR block, convolved 388 with the canonical HRF, for each BR condition. A GLM was performed at the 389 single participant level. Beta values for each of the trained ROls were 390 averaged for each condition and adjusted for the global brain signal. Mean 391 percentage signal change (PSC) was then calculated.

392

393 For inferential statistical analyses, SPSS 21 (IBM Corp. Armonk, USA) was 394 used to perform ANOVAs and follow-up planned paired sample t-tests, which 395 were two-tailed unless otherwise stated.

\section{Control Experiment- Mental Imagery:}

\section{Experimental outline}

402 Ten different participants (age range $=22-39$ years, mean age 30 . years, 8 403 females) were recruited for a control BR experiment. They viewed a Dell LCD 404 monitor (width: $43.5 \mathrm{~cm}$; resolution: 1600 900; refresh rate: $60 \mathrm{~Hz}$ ) from a 405 distance of $43 \mathrm{~cm}$ (fixed using a chin rest) through a mirror stereoscope. The 406 stereoscope reflected the left and right sides of the screen into the 
participants' left and right eyes, so that each eye was presented with only one

408 of the two images (house or face). In order to ensure robust fusion of 409 binocular images, prior to the start of BR task, fusion was achieved for each 410 participant by slowly moving two grey squares from the edge toward the 411 centre of the screen. At the beginning of this process the participants would 412 see two squares. By the end of this process the participants would report 413 when they were seeing one square. All testing took place in a darkened room. 414 During the viewing blocks, a face stimulus and a house stimulus were 415 presented in the left and right hemi-fields respectively. The stimuli were 416 pseudorandomised with regards to which eye received the face or house 417 stimuli. Each viewing block (40 s followed by rest $20 \mathrm{~s}$ ) was performed with a 418 new pair of stimuli from the pool of twenty stimuli. Six blocks were performed 419 per session, for three sessions. Participants were instructed to indicate a 420 perceptual shift only if the whole exemplar was perceived; any combination or 421 'patchwork' percept regardless of the predominance of the exemplar category 422 was reported as a 'mixed' percept. The participants were instructed to switch 423 as accurately and rapidly as possible between three possible button presses 424 linked to the three perceptual states (face percept, house percept, mixed 425 percept). This resulted in measures of the cumulative duration of the percept 426 throughout the BR measurement period.

428 BR was performed in this manner prior to and after 3 days of consecutive 429 mental imagery training (see below). 
433 Participants returned to perform mental imagery training. Participants were 434 pseudorandomised into two equal groups, and were explicitly advised to use 435 mental imagery strategies that involved faces ('Face group') or house/places 436 ('House group'). Mental imagery was undertaken while viewing a LCD monitor 437 screen with a fixed horizontal black bar. They were told to imagine pushing a 438 bar above the fixed black bar, while performing their mental imagery 439 strategies. Each mental imagery training session comprised three sessions, 440 each including six blocks of $60 \mathrm{~s}$ with a 'perform mental imagery' period (40 s) 441 followed by 'rest' (20 s).

\section{Brain Structural Analysis}

445 The structural analysis was performed using Tensor Based Morphometry 446 (TBM), an emerging computational analysis technique(Ceccarelli et al., 2009; 447 Farbota et al., 2012; Li et al., 2009; Wang et al., 2013; Welch et al., 2013), 448 which is better suited to studies with smaller participant samples. TBM 449 enables longitudinal quantitative assessment by identifying regional structural 450 differences from the gradients of the deformation fields that nonlinearly warp 451 each individual image to the template.

453 For each participant, high-resolution T1 structural images were reoriented 454 placing the anterior commissure at the MNI origin. Longitudinal nonlinear 455 registration(Ashburner and Ridgway, 2012) was performed to align the two 456 time-points (before and after training) to their within-subject average, 
457 characterising the relative volumetric expansion or contraction (as the 458 divergence of a velocity field) of each voxel in each time-point with respect to

459 the average. The within-subject average images were then segmented to 460 produce grey and white matter segmentations for each participant(Ashburner 461 and Friston, 2005). These segmentations were nonlinearly aligned to their 462 group-wise average using Dartel (Ashburner, 2007), and the final Dartel 463 average template was affinely registered to $\mathrm{MNI}$ space. The resultant 464 between-subject transformations were then used to spatially normalise the 465 divergence maps of the velocity fields, which were finally smoothed with a 466 6mm FWHM Gaussian kernel.

468 Divergence measures for each participant were then extracted within 469 spherical ROls for FFA and PPA $(6 \mathrm{~mm})$. The spheres were centered on 470 coordinates that demonstrated the highest functional activity within the 471 localiser ROls across training. A t-test was then performed to establish if a 472 specific brain region had changed significantly before versus after training.

\section{Canonical Variate Analysis}

476 We used a Canonical Variate Analysis (CVA) to demonstrate that measures 477 of change in brain activation and brain structure following neurofeedback 478 training predicted changes in behavioural measures. Also known as a 479 multivariate analysis of variance, or ManCova (Friston et al., 2014, 1995), 480 CVA enables statistical inferences to be made about associations between 481 the imaging data, and behavioural data that are distributed over variables. It 
was chosen for analysis of this dataset because it can accommodate 483 statistical dependencies between multivariate predictor variables (behavioural 484 changes) and multivariate outcome variables (functional or structural 485 measures). Neither the behavioural nor imaging data had to be examined in 486 isolation, which had the advantage that distributed changes could be 487 identified, while minimising the multiple comparisons problem. The 488 behavioural changes for each participant was the change in dominance 489 duration of each the three percepts (e.g. strategy-related percept, strategy490 unrelated percept, mixed percept) between the pre-training BR condition and 491 post-training BR (Figure S3), and between the pre-training BR condition and 492 post-training BR with concurrent trained up-regulation (Figure S3). As the 493 behavioural and structural measures were taken prior to and immediately after 494 neurofeedback training, the functional measures for each participant were the 495 change in the different signal between the first and the last training run (e.g. 496 run 1 and run 9). The structural measures for each participant were the 497 divergence measures for each ROI, FFA and PPA (6 mm).

499 The objective of the CVA was to find the linear combination of outcome 500 variables that was best predicted by a linear mixture (contrast) of structural or 501 functional components. The weights of these linear combinations are called 502 canonical vectors. The canonical variates of the outcome and predictor 503 variables are the expression of each canonical vector in each subject. Other 504 quantities generated by the CVA include Bartlett's approximate chi-squared 505 statistic for Wilks' Lambda and its associated significance, or p-value, which 506 test for the significance of a linear mapping or correlation between the 
507 canonical variates (in other words, if one or more pairs of canonical variates 508 show a significant statistical dependency).

509

510

511

512 


\section{Results}

514

515 We first examined the effect of neurofeedback training on behaviour using

516 three comparisons. We compared perceptual dynamics, specifically

517 cumulative dominance durations, performed during BR before and after

518 training. We then examined the effects of learned upregulation on BR by

519 comparing pre-training BR versus post-training BR with concurrent 'learned'

520 upregulation of brain activity. For the final comparison, we examined the non-

521 trained mental imagery on BR, by comparing perceptual dynamics during pre-

522 training BR versus post-training BR with concurrent non-trained mental

523 imagery. The effects of trained mental imagery were additionally examined

524 separately - see Mental Imagery Control Experiment and Figure S1

525 (Supplementary Materials).

526

527 As the durations of the three percepts were dependent on each other, a

528 change in one percept occurred linked to changes in one or both of the other

529 percepts.

530

531 Within Condition Comparisons:

532 Comparison 1. Pre-training BR vs. Post-training BR

533 Comparing behavioural measures of pre-training BR and post-training BR

534 indicated an effect of training (Figure 3). Paired t-tests revealed a significant 535 reduction in the cumulative dominance durations (i.e. how long a percept type 536 was perceived) for the strategy-unrelated percept $(t(9)=2.88, p=0.02)$, and a 537 significant increase in mixed percept durations $(t(9)=2.74, p=0.02)$, with no 
538 significant change in the dominance duration of the strategy-related percept

$539(\mathrm{t}(9)=0.46, \mathrm{p}=0.66)$.

540

541 Comparison 2. Pre-training BR vs. Post-training BR with Concurrent

542 Trained Upregulation

543 Paired t-tests revealed a significant reduction in the duration of the strategy-

544 unrelated percept $(\mathrm{t}(9)=4.76, \mathrm{p}=0.001)$, and a significant increase in the

545 duration of the mixed percept $(t(9)=2.68, p=0.03)$. There was no significant

546 change in the dominance duration of the strategy-related percept

$547(\mathrm{t}(9)=0.53, \mathrm{p}=0.61)$ (Figure 3$)$. The changes in BR dynamics were similar to

548 those observed with pre-training BR vs. post-training BR (Comparison 1).

549

550 Comparison 3. Pre-training BR vs. Post-training BR with concurrent

$551 \quad$ Non-trained Mental Imagery

552 Paired t-tests indicated a significant reduction in the duration of the strategy-

553 related percept $(\mathrm{t}(9)=2.41, \mathrm{p}=0.04)$, and a significant increase in the duration

554 of the mixed percept $(\mathrm{t}(9)=2.68, \mathrm{p}=0.03)$. There was no significant change in

555 the dominance duration of the strategy-unrelated percept $(t(9)=0.12, p=1.74)$.

556

557 We further examined differences between conditions.

558

559 Between Condition Comparisons:

560

561 1. Post-training BR vs. Post-training BR with Concurrent Trained

562 Upregulation (Comparison 1 vs. Comparison 2) 
563 There was a significantly greater reduction in the dominance duration of the

564 strategy-unrelated percept $(\mathrm{t}(9)=2.40, \mathrm{p}=0.04)$ in Comparison 2 as compared

565 with Comparison 1 (Figure 3B). There were no other significant differences

566 between the two comparisons (strategy-related percept: $t(9)=0.95, p=0.37$;

567 mixed percept: $\mathrm{t}(9)=0.90, \mathrm{p}=0.39)$.

568

569

2. Post-training BR vs. Post-training BR with Non-trained Mental Imagery

(Comparison 1 vs. Comparison 3)

571 There was a significantly greater reduction in the dominance duration of the

572 strategy-related percept $(\mathrm{t}(9)=3.12, \mathrm{p}=0.01$ ) (Figure $3 \mathrm{~B})$. There was also a

573 significantly greater increase in the dominance duration of the mixed percept

$574(\mathrm{t}(9)=2.62, \mathrm{p}=0.03)$. There were no significant changes in the strategy-

575 unrelated percept $(\mathrm{t}(9)=0.09, \mathrm{p}=0.93)$

576

577 3. Post-training BR with Concurrent Trained Upregulation vs. Post-

578 training BR with Non-trained Mental Imagery (Comparison 2 vs.

579 Comparison 3)

580 There was a trend towards reduction in the dominance duration of the

581 strategy-related percept $(\mathrm{t}(9)=2.23, \mathrm{p}=0.05)$ in Comparison 3 as compared

582 with Comparison 2 (Figure 3B). The other two comparisons were not

583 significant (strategy-related percept: $t(9)=0.95, \quad p=0.37$; mixed percept:

$584 t(9)=1.1, p=0.30)$.

585

586 Functional Changes during Neurofeedback Training 
587 To test if neurofeedback training resulted in progressive learning, we 588 examined whether participants demonstrated increased control of the

589 differential feedback signal over the three training days (Figure 2B). A 590 repeated-measures ANOVA with a factor of training day (3 levels; Days 1-3)

591 demonstrated a significant effect $(F(2,16)=3.74, p=0.047)$. Post-hoc t-tests 592 demonstrated a significant increase in the differential signal from Day 2 593 onwards, suggesting a learning effect (Day 1: $t(9)=0.88, p=0.40$; Day 2: $594 \mathrm{t}(9)=3.27 \mathrm{p}=0.001 ;$ Day $3: \mathrm{t}(8)=2.75, \mathrm{p}=0.02)$.

595

596 Functional Changes during Transfer

597 Following neurofeedback training and prior to BR, voluntary control of brain 598 activation in the absence of neurofeedback was confirmed in a 'transfer 599 session'. Differential BOLD activation (strategy-related ROI minus strategy600 unrelated ROI) pooled across the two transfer sessions, revealed a significant 601 effect $(t(9)=2.38, p=0.04)$.

602

\section{Functional Changes during Binocular Rivalry}

604 We examined task-related BOLD signals in the trained ROIs (FFA and PPA) 605 comparing pre-training BR with post-training BR. We observed significant 606 reductions in BOLD signals in both the strategy-related $\mathrm{ROI}(\mathrm{t}(9)=3.43, \mathrm{p}=$ 607 0.007) and strategy-unrelated $\mathrm{ROI}(\mathrm{t}(9)=2.26, \mathrm{p}=0.04)$, when comparing pre608 training BR with post-training BR.

609

610 Comparing pre-training BR versus post-training BR with concurrent trained 611 upregulation, there was a significant reduction in the activation level of the 
612 strategy-unrelated $\mathrm{ROI}(\mathrm{t}(9)=2.48, \mathrm{p}=0.03)$. No significant change was noted

613 for the strategy-related $\mathrm{ROI}(\mathrm{t}(9)=1.41, \mathrm{p}=0.19)$. We performed one-tailed $\mathrm{t}-$

614 tests as we had an a priori hypothesis that following neurofeedback training,

615 participants should be able to increase the difference in BOLD activation

616 between the two trained ROls (Figure 4).

617

618 There were no significant changes for pre-training BR versus post-training BR

619 with concurrent non-trained mental imagery (strategy related $\mathrm{ROI}: \mathrm{t}(9)=0.82$,

$620 \mathrm{p}=0.44$; strategy unrelated ROI: $\mathrm{t}(9)=0.83, \mathrm{p}=0.43)$.

621

622 Mental Imagery Control Experiment

623

624 There was no evidence of significant changes in the cumulative dominance 625 durations of any of the three percepts (strategy-related percept $t(9)=0.74, p=$ 626 0.48; strategy-unrelated percept, $t(9)=1.00, p=0.34$; mixed percept, $t(9)=$ 627 2.00, $p=0.07)$

628

629

Between Group Comparisons with ‘Mental Imagery’ Control Group

630 We performed an ANOVA with a within-subjects factor of percepts (strategy-

631 related percept, strategy-unrelated percept, mixed percept)) and a between-

632 subjects factor of group (Group 1: neurofeedback, Group 2: mental imagery).

633 There was a main effect of percept $(F(2,36)=4.64, p=0.02)$. There was no

634 interaction $(F(2,36)=2.65, p=0.08)$ between these two factors.

635 
636 We performed a second ANOVA with a within-subjects factor of percepts 637 (strategy-related percept, strategy-unrelated percept, mixed percept)) and a 638 between-subjects factor of group (Group 1: neurofeedback with concurrent639 upregulation, Group 2: mental imagery). There was a main effect of percept $640(\mathrm{~F}(2,36)=6.68, \mathrm{p}=0.003)$, and an interaction between percept and group $641(F(2,36)=5.29, p=0.01)$. Follow-up two-sample t-tests looking at changes in 642 durations of the similar percepts showed a significant difference for the 643 strategy-unrelated percept $(t(9)=2.35, p=0.04)$, but not for strategy-related 644 percept $(t(9)=1.04, p=0.32)$ or the mixed percept, $(t(9)=2.00, p=0.08)$.

\section{5}

646 We performed a further ANOVA with a within-subjects factor of percepts 647 (strategy-related percept, strategy-unrelated percept, mixed percept)) and a 648 between-subjects factor of group (Group 1: neurofeedback with concurrent 649 non-trained mental imagery, Group 2: mental imagery). There was a main 650 effect of percept $(F(2,36)=6.70, p=0.003)$, and an interaction between 651 percept and group $(\mathrm{F}(2,36)=3.63, \mathrm{p}=0.04)$. Follow-up two-sample t-tests 652 looking at changes in durations of the similar percepts showed a significant 653 difference for the mixed percept $(t(9)=2.79, p=0.02$, but not for strategy654 related percept $(\mathrm{t}(9)=1.00, \mathrm{p}=0.86)$ or the strategy-unrelated percept, $(\mathrm{t}(9)=$ $6550.29, p=1.14)$ 
661 The results of the longitudinal non-rigid registration were used to determine 662 volume changes in the ROls by calculating the_divergence of the velocity 663 fields. One-sampled t-tests of these values were used to calculate if any 664 significant structural changes had taken place as a result of neurofeedback 665 training. They were not significant for both ROls i.e. FFA $(t(9)=0.36, p>0.05)$, 666 and PPA $(t(9)=0.46, p>0.05)$,

\section{Results - Canonical Variate analysis}

670 Plots for comparisons of combined measures in: (1) behaviour (dominance

671 durations for the three perceptual reports) and functional (BOLD changes 672 across training in FFA, PPA); and (2) behaviour and structural measures 673 (measure of the volume changes in FFA and PPA following training) are 674 presented in Figure S4, together with Bartlett's approximate chi-squared 675 statistic for Wilks' Lambda and its p-value, for each comparison.

677 The participant neurofeedback training measures (i.e. differential BOLD brain 678 activation) had a trend to being correlated with changes in BR behavioural 679 dynamics as recorded during BR with concurrent trained upregulation of brain 680 activation (compared with pre-training $B R$ ) (chi-squared value $=12.35, p=$ 681 0.05). Comparison of changes in the neurofeedback training measures with 682 behavioural changes during 'simple BR' before and after training was non683 significant (chi-square value $=11.43, p=0.07$ ). Significant correlations were 
684 noted between structural changes in both ROls and the change in BR 685 dynamics produced during concurrent trained upregulation of brain activation 686 (chi-squared value $=19.64, \mathrm{p}=0.03$ ). Comparison of structural measures with 687 behavioural measures during 'simple BR' before and after training was non688 significant (chi-square value $=13.77, \mathrm{p}=0.09$ ).

689

690 Of note, the mapping weights obtained for the behavioural measures and the 691 training-related BOLD measures were independent of the mapping weights 692 obtained for the behavioural measures and the structural measures. This is 693 because these multivariate mapping values were specific to the measures 694 used in the comparisons. Finally, the interpretation of the mapping weights in 695 relation to having a positive or negative value did not indicate a positive or 696 negative change in the values (e.g. an increase or decrease in structural 697 measures). Rather they represent a positive (or negative) contribution to the 698 mapping between the multivariate predictor variable and the outcome 699 variables. 


\section{Discussion}

704 Participants learned to differentially regulate the amplitude of BOLD activation 705 in two higher-order visual brain regions, FFA and PPA. This was achieved in 706 realtime, through volitional control using neurofeedback training with rt-fMRI. 707 The use of a 'differential training signal was implemented by showing the 708 participants a 'thermometer bar' whose size represented the difference in the 709 mean BOLD signal between the two selected brain regions. By doing this, one 710 of the brain regions acted as an internal control for the other, accounting for

711 potential confounds produced by global changes in brain activation in 712 response to effects such as arousal. Furthermore, specific behavioural effects 713 linked with the direction of change of the differential training signal were 714 obtained, providing a comparison of behavioural metrics for the training 715 effect(Thibault et al., 2018). The effect on visual perception was examined 716 with an independent BR task that employed stimuli specifically engaging 717 these stimulus-selective brain regions (face stimuli for FFA, house stimuli for 718 PPA). During BR, moment-to-moment stochastic alternations between two 719 competing visual percepts are observed, while concurrent brain activity can 720 be recorded and potentially manipulated(Blake et al., 2014; Blake and 721 Logothetis, 2002).

723 In this study, a change in BR perceptual dynamics was observed following 724 neurofeedback training. Perception of the stimulus linked to neurofeedback 725 training was rendered more stable e.g. strategy-related percept, with a 726 reduction in the perception of the other stimulus e.g. strategy-unrelated 
percept. This behavioural change occurred when comparing pre-training BR

728 with post-training BR, and additionally when participants performed post-

729 training BR while concurrently performing learned 'upregulation' of brain

730 activity. We compared pre-training 'BR' with three post-training BR conditions:

731 'post-training BR', 'post-training BR with concurrent trained upregulation', and

732 'post-training BR with concurrent non-trained mental imagery'. The first

733 comparison, examining changes during BR before and after neurofeedback

734 training, showed altered BR dynamics; specifically a reduction in the

735 cumulative dominance duration of the strategy-unrelated percept. These

736 findings are important, as they show that neurofeedback training produced a

737 behavioural effect that was: (1) counter-intuitive in that percept durations were

738 not increased in line with the verbally instructed neurofeedback training

739 strategy, which was initially expected. Rather, percept durations not linked to

740 the neurofeedback training strategy (e.g. strategy-unrelated percept) were

741 reduced; (2) aligned with a longstanding finding in the field, namely Levelt's

742 second proposition (discussed below); and (3) indicative of a lack of demand

743 characteristics (see also Mental Imagery Control Experiment).

744

745 There was a significant reduction in the levels of activation in both ROls,

746 comparing pre-training BR versus post-training BR. This linked neuroimaging

747 finding was unexpected, as the prediction from existing literature(Tong et al.,

748 1998) is that BOLD activation levels in extrastriate visual areas will reflect

749 dominance durations. The expected finding might have been that activation

750 levels would be lower in the strategy-unrelated ROI. Our findings instead

751 showed that both regions were affected by neurofeedback training, as we 
expected given that participants trained on a differential signal involving both

753 ROIs. Both ROls demonstrated a reduction in activation, which may reflect an

754 increase in neural efficiency as a result of more precise tuning of neural 755 representations(Gimenez et al., 2014; Haler et al., 1992; Heinzel et al., 2014;

756 Vartanian et al., 2013). The exact mechanisms underlying this gain are 757 unknown, particularly in the context of neurofeedback training and thus 758 warrants further study(Poldrack, 2015).

760 The purpose of the second comparison ('pre-training BR' versus 'post-training 761 BR with concurrent upregulation') was to examine if there was an effect of 762 concurrent trained modulation of brain activation on BR dynamics that was 763 additive or different to the effect of neurofeedback training alone. We 764 observed a change in BR dynamics that was similar and greater to that 765 observed for pre-training BR vs. post-training BR, in that there was more of a 766 reduction in the mean dominance duration of the strategy-unrelated percept. 767 This confirmed that the effect of trained upregulation was directly aligned with 768 the effect of neurofeedback training on BR dynamics. There was a decrease 769 in the level of BOLD activation in the strategy-unrelated ROI only, with no 770 significant change in the strategy-related ROI. Interestingly, these BOLD 771 activation changes were the same as those observed during neurofeedback 772 training (a reduction in activation levels of the strategy-unrelated ROI, Figure 773 2B). This provides further evidence for a similar mechanism underlying the 774 changes in BR dynamics following training and for those observed with 775 concurrent trained upregulation. The counter-intuitive effect of training and up776 regulation (during $\mathrm{BR}$ ) on the brain activations in the two ROls (i.e. opposite to 
an a priori instruction and predicted direction of activation changes) is

778 intriguing and worthy of further investigation(Abel et al., 2015; Bueichekú et

779 al., 2016).

780 The third comparison ('pre-training BR' versus 'post-training BR with non-

781 trained mental imagery') served to assess the impact of using a differential

782 training signal, which was hypothesised to have an effect on both ROls in all

783 participants. It additionally helped reveal the role of non-trained mental

784 imagery in the context of prior neurofeedback training. No significant change

785 in brain activation in either ROI was observed. However, BR dynamics

786 changed in a similar manner to the other two post-neurofeedback training BR

787 conditions, with a significant reduction in the duration of the percept not linked

788 to the training strategy used during training. This reduction was significant

789 when comparing changes in perceptual dynamics across conditions. These

790 behavioural findings would therefore suggest that neurofeedback training,

791 despite the lack of a statistically significant BOLD effect, produced a more

792 general effect on the neurobiology of the two trained ROls. The exact nature

793 of this effect may be complex, given that behavioural changes observed for

794 this condition were opposite to the direction of neurofeedback training, but

795 nonetheless sufficient to produce an effect e.g. 'House' group participants

796 specifically underwent neurofeedback training with 'House-based' mental

797 imagery strategies, and yet they generated changes in BR dynamics simply

798 by using non-trained 'face' based mental imagery strategies during the

799 performance of BR. These behavioural findings are different from Rademaker

800 and Pearson's work, in which using mental imagery training did not produce

801 training-related changes in BR dominance duration. Five successive days of 
mental imagery training had no effect on BR, with no benefit being conferred by expending increased effort during mental imagery generation(Rademaker

804 and Pearson, 2012). On the other hand, Rademaker and Pearson's findings

805 are in keeping with our own mental imagery control experiment, indicating the

806 relevance of neurofeedback training. We conducted a behavioural control

807 experiment in which a different group of participants performed BR before and

808 after three consecutive days of mental imagery training, which was analogous

809 to the neurofeedback training. The training was again explicitly linked to one

810 of the two stimuli used in BR (face mental imagery for a 'Face group', house

811 mental imagery for a 'House group'). However there was no targeted training

812 strategy for the brain, unlike with the neurofeedback-trained groups. No

813 significant changes in dominance durations of any of the three percepts were

814 observed.

815

816 Taken together, these results indicate that short-term intensive training over 3

817 days on a neurofeedback BOLD signal produced by two brain regions, 818 engages and alters the function and biology of both regions. This is 819 specifically supported by the shift in perceptual dynamics during BR following 820 neurofeedback training, and the activation changes observed in both ROls

821 (see Results: Comparison 1). It is further supported more broadly by the 822 behavioural changes observed in all of the post-neurofeedback training BR 823 conditions, which were not observed in the mental imagery control 824 experiment. Habes et al.(Habes et al., 2016) have previously confirmed that 825 although differential regulation of category-specific visual areas can be 826 achieved after a single day of training, a linked change in BR dynamics was 
827 not produced. We therefore suggest that in order for mental imagery to 828 produce a change in perception, it must be linked with neurofeedback-led 829 learning, conducted over a period of days. This may be attributable to the 830 interposition of sleep with sequential daily training. Sleep has been directly 831 linked with the offline processing necessary for the consolidation of 832 neuroprosthetic learning(Gulati et al., 2014) and associated behavioural 833 output(Gulati et al., 2017).

834

835 Mental imagery may be utilised for perceptual learning of low-level visual 836 features, and to activate stimulus-selective cortical representations(O'Craven 837 and Kanwisher, 2000; Tartaglia et al., 2009). Similarly, rt-fMRI neurofeedback 838 together with implicit operant reinforcement has been used to unconsciously 839 train patterns of activation in primary visual brain regions(Amano et al., 2016; 840 Shibata et al., 2011) to produce perceptual and associative learning of low841 level visual features such as colour and orientation. However, to-date neither 842 approach has successfully yielded changes in higher-order visual perception.

843 In this study, we show that coupling explicitly instructed mental imagery with 844 rt-fMRI neurofeedback training of higher-order visual brain regions produces 845 an unconscious and targeted shift in the perceptual processing of visual 846 stimuli. This result is novel and significant in providing evidence for non847 invasively manipulating higher-order brain function, potentially at the level of 848 directly strengthening neural representations to alter higher-order 849 perception(Fahle, 2002; Watanabe et al., 2002, 2001). From a mechanistic 850 perspective, an interesting next step might be to test if unconsciously inducing 851 specific patterns of brain activations related to category-specific stimuli will 
852 produce linked shifts in perception in a similar manner to that observed in this 853 study(Watanabe et al., 2017). This would provide more direct evidence of

854 modulating neural representations.

856 The observed behavioural findings may constitute a neural analogue of 857 Levelt's second proposition(Levelt, 1966), as applied to stimulus perception. 858 The original proposition (see Supplementary Discussion) was based on the 859 physical properties of visual stimuli. It was recently modified to indicate that 860 'increasing the difference in stimulus strength between the two eyes will 861 primarily act to increase the average perceptual dominance duration of the 862 stronger stimulus'(Brascamp et al., 2015). Our work may provide evidence for 863 a neural reformulation of BR. Participants were trained on a differential signal, 864 rather than specifically training to increase the level of activation in the 865 strategy-related ROI. During training, they appeared to reduce the level of 866 activation in the strategy-unrelated ROI across the three days, while 867 maintaining a fixed level of activation in the strategy-related ROI (Figure 2B). 868 This difference in activation levels as a result of training was maintained when 869 the participants undertook the transfer sessions, an assessment of 870 upregulation in the absence of neurofeedback. The difference in $\mathrm{ROI}$ 871 activation levels may have therefore led to a relative difference in the 872 strengths of the neural representations linked to the visual stimulus 873 categories. In keeping with this view, we observed a reduction in the mean 874 dominance duration of the strategy-unrelated percept. This resulted in greater 875 mean dominance durations of the strategy-related percept, corresponding to 876 the ROI with the strengthened neural representation. On the basis of this, we 
877 propose a possible neural analogue of the Levelt's modified second 878 proposition as follows: 'increasing the difference in neural representation 879 strengths between the two brain regions linked to the two monocular visual 880 stimuli will primarily act to increase the average perceptual dominance of the 881 percept linked to the stronger neural representation'. The effect of this would 882 be to produce unconscious perceptual biasing towards the strengthened 883 percept. This mechanism for perceptual 'shaping'(Lange et al., 2018) may 884 have real-world application in conditions requiring targeted enhancement of 885 perception such as in threat detection(Miranda et al., 2015), or therapeutically 886 to reduce unwanted or aberrant percepts(Taschereau-Dumouchel et al., 887 2018).

889 Several mechanisms have been put forward to explain the neural 890 underpinnings of BR. Of note, known influences on visual perception such as 891 priming and cueing have not been shown to produce changes in BR 892 dominance durations (see also Supplementary Discussion). Neurofeedback 893 with rt-fMRI provides the most direct means of testing neuronal function 894 involved in processing visual stimuli. Using a hierarchical model of BR(Dayan, 895 1998), it may be proposed that neurofeedback training of higher order brain 896 regions strengthens neuronal representations linked to the processing of 897 specific visual stimuli, leading to unconscious perceptual biasing. Preferential 898 processing of strategy-related stimuli would result in decreased dominance 899 durations of the strategy-unrelated stimuli, as was observed here. The effect 900 of neurofeedback on BR may be further considered within a Bayesian 901 framework(Lange et al., 2018). During BR, the dominant percept at any given 
time is maintained by the highest posterior probability, at the top of the cortical

903 hierarchy. Stimulus representations at lower levels generate error signals that

904 are compared with top-down predictions. The percept is rendered more or

905 less stable in relation to bottom-up inhibition i.e. the lower the error signal, the

906 more stable the percept(Alink et al., 2010; Hohwy et al., 2008; Summerfield

907 and Koechlin, 2008). In keeping with this, BR dynamics were shifted in the

908 direction of the information represented in the trained visual brain regions.

909 Therefore, perception of the stimulus linked to training was rendered more

910 stable, with a simultaneous reduction in the stability of the perception of the

911 other stimulus, leading to a reduction in its mean dominance duration.

912 The changes in high-level visual perception following neurofeedback training

913 in this study were associated with structural changes in the trained regions

914 (see Supplementary Materials). We used a multivariate analysis technique,

915 Canonical Variate Analysis, which can accommodate multiple measures of

916 behaviour, structure, and function to help determine the overarching effect of

917 neurofeedback training. The change in BR dynamics (i.e. cumulative

918 dominance durations) was linked with measures of structural changes in FFA,

919 and PPA (Figure S3, Supplementary Materials). These preliminary findings in

920 ten participants suggest that neurofeedback training, even over a relatively

921 short period of time (3 days) can alter perception as a result of plasticity in the

922 trained brain regions(Johansen-Berg et al., 2012; Sagi et al., 2012).

923

924 In this study, we provide a direct demonstration of the rapid changes in 925 perception and neural plasticity that can be produced by neurofeedback 926 training of higher-order visual areas using rt-fMRI. Imagery-related activation 
927 in higher-order visual cortex, such as the ventral visual areas, are related to 928 semantic content, and are more flexible and abstract(Orban et al., 2014) as 929 compared to early visual cortex. Therefore, the use of higher-order visual 930 areas paired with rt-fMRI neurofeedback training may provide the most potent 931 and generalizable means of enacting a change on complex perception. Neural 932 representations that give rise to prior expectations can be directly shifted in 933 the direction of neurofeedback training, even in the presence of pre-existing 934 expectations. This could lead to targeted enhancement of specific responses 935 during discrete tasks as demonstrated here using BR, or in the reduction of 936 aberrant visual perception, such as hallucinations, for therapeutic 937 effect(Lange et al., 2018).

938 
939

940

941

942

943

944

945

946

947

948

949

950

951

952

953

954

955

956

957

958

959

960

961

962

963

964

\section{References}

Abel, S., Weiller, C., Huber, W., Willmes, K., Specht, K., 2015. Therapyinduced brain reorganization patterns in aphasia. Brain 138, 1097-1112. doi:10.1093/brain/awv022

Alink, A., Schwiedrzik, C.M., Kohler, A., Singer, W., Muckli, L., 2010. Stimulus predictability reduces responses in primary visual cortex. J. Neurosci. 30, 2960-6. doi:10.1523/JNEUROSCI.3730-10.2010

Amano, K., Shibata, K., Kawato, M., Sasaki, Y., Watanabe, T., 2016. Learning to Associate Orientation with Color in Early Visual Areas by Associative Decoded fMRI Neurofeedback. Curr. Biol. 26, 1861-1866. doi:10.1016/j.cub.2016.05.014

Ashburner, J., 2007. A fast diffeomorphic image registration algorithm. Neuroimage 38, 95-113. doi:10.1016/j.neuroimage.2007.07.007

Ashburner, J., Friston, K.J., 2005. Unified segmentation. Neuroimage 26, 839-51. doi:10.1016/j.neuroimage.2005.02.018

Ashburner, J., Ridgway, G.R., 2012. Symmetric diffeomorphic modeling of longitudinal structural MRI. Front. Neurosci. 6, 197. doi:10.3389/fnins.2012.00197

Blake, R., Brascamp, J., Heeger, D.J., Brascamp, J., 2014. Can binocular rivalry reveal neural correlates of consciousness?

Blake, R., Logothetis, N.K., 2002. Visual competition. Nat. Rev. Neurosci. 3, 13-21. doi:10.1038/nrn701

Blefari, M.L., Sulzer, J., Hepp-Reymond, M.-C., Kollias, S., Gassert, R., 2015. Improvement in precision grip force control with self-modulation of 
primary motor cortex during motor imagery. Front. Behav. Neurosci. 9, 18. doi:10.3389/fnbeh.2015.00018

967

Brascamp, J.W., Klink, P.C., Levelt, W.J.M., 2015. The 'laws' of binocular rivalry: 50 years of Levelt's propositions. Vision Res. 109, 20-37.

969 doi:10.1016/j.visres.2015.02.019

970

Bueichekú, E., Miró-Padilla, A., Palomar-García, M.-Á., Ventura-Campos, N., Parcet, M.-A., Barrós-Loscertales, A., Ávila, C., 2016. Reduced posterior parietal cortex activation after training on a visual search task. Neuroimage 135, 204-213. doi:10.1016/j.neuroimage.2016.04.059

974 Ceccarelli, A., Rocca, M.A., Pagani, E., Falini, A., Comi, G., Filippi, M., 2009. Cognitive learning is associated with gray matter changes in healthy human individuals: a tensor-based morphometry study. Neuroimage 48, 585-9. doi:10.1016/j.neuroimage.2009.07.009

978 Cordes, D., Haughton, V.M., Arfanakis, K., Carew, J.D., Turski, P.A., Moritz, 979 C.H., Quigley, M.A., Meyerand, M.E., 2001. Frequencies Contributing to 980 Functional Connectivity in the Cerebral Cortex in "Resting-state" Data. $981 \quad$ AJNR Am. J. Neuroradiol. 22, 1326-1333.

982 Dayan, P., 1998. A hierarchical model of binocular rivalry. Neural Comput. 10, 983 1119-35.

984 deCharms, R.C., Christoff, K., Glover, G.H., Pauly, J.M., Whitfield, S., 985 Gabrieli, J.D.., 2004. Learned regulation of spatially localized brain 986 987 activation using real-time fMRI. Neuroimage 21, 436-443.

988 Deichmann, R., Schwarzbauer, C., Turner, R., 2004. Optimisation of the 3D 989 MDEFT sequence for anatomical brain imaging: technical implications at 
992 Eickhoff, S.B., Heim, S., Zilles, K., Amunts, K., 2006. Testing anatomically specified hypotheses in functional imaging using cytoarchitectonic maps, Neurolmage. doi:10.1016/j.neuroimage.2006.04.204

995 Eickhoff, S.B., Stephan, K.E., Mohlberg, H., Grefkes, C., Fink, G.R., Amunts, K., Zilles, K., 2005. A new SPM toolbox for combining probabilistic cytoarchitectonic maps and functional imaging data, Neurolmage. doi:10.1016/j.neuroimage.2004.12.034

Epstein, R., Kanwisher, N., 1998. A cortical representation of the local visual 1000 environment. Nature 392, 598-601. doi:10.1038/33402

1001 Fahle, M., 2002. Perceptual learning: gain without pain? Nat. Neurosci. 5, 1002 923-924. doi:10.1038/nn1002-923

1003 Farbota, K.D.M., Sodhi, A., Bendlin, B.B., McLaren, D.G., Xu, G., Rowley, H. 1004 a, Johnson, S.C., 2012. Longitudinal volumetric changes following 1005 traumatic brain injury: a tensor-based morphometry study. J. Int. 1006 Neuropsychol. Soc. 18, 1006-18. doi:10.1017/S1355617712000835

1007 Friston, K.J., Frith, C.D., Frackowiak, R.S.J., Turner, R., 1995. Characterizing 1008 Dynamic Brain Responses with fMRI: A Multivariate Approach. $1009 \quad$ Neuroimage 2, 166-172. doi:10.1006/nimg.1995.1019 1010 Friston, K.J., Kahan, J., Biswal, B., Razi, A., 2014. A DCM for resting state 1011 fMRI. Neuroimage 94, 396-407. doi:10.1016/j.neuroimage.2013.12.009 1012 Gimenez, P., Bugescu, N., Black, J.M., Hancock, R., Pugh, K., Nagamine, M., 1013 Kutner, E., Mazaika, P., Hendren, R., McCandliss, B.D., Hoeft, F., 2014. 1014 Neuroimaging correlates of handwriting quality as children learn to read 
1015

1016

1017

1018

1019

1020

1021

1022

1023

1024

1025

1026

1027

1028

1029

1030

1031

1032

1033

1034

1035

1036

1037

1038

1039

and write. Front. Hum. Neurosci. 8, 155. doi:10.3389/fnhum.2014.00155

Goebel, R., Esposito, F., Formisano, E., 2006. Analysis of functional image analysis contest (FIAC) data with brainvoyager QX: From single-subject to cortically aligned group general linear model analysis and selforganizing group independent component analysis. Hum. Brain Mapp. 27, 392-401. doi:10.1002/hbm.20249

Gulati, T., Guo, L., Ramanathan, D.S., Bodepudi, A., Ganguly, K., 2017. Neural reactivations during sleep determine network credit assignment. Nat. Neurosci. 20, 1277-1284. doi:10.1038/nn.4601

Gulati, T., Ramanathan, D.S., Wong, C.C., Ganguly, K., 2014. Reactivation of emergent task-related ensembles during slow-wave sleep after neuroprosthetic learning. Nat. Neurosci. 17, 1107-13. doi:10.1038/nn.3759

Habes, I., Rushton, S., Johnston, S.J., Sokunbi, M.O., Barawi, K., Brosnan, M., Daly, T., Ihssen, N., Linden, D.E.J., 2016. fMRI neurofeedback of higher visual areas and perceptual biases. Neuropsychologia 85, 208215. doi:10.1016/j.neuropsychologia.2016.03.031

Haler, R.J., Siegel, B., Tang, C., Abel, L., Buchsbaum, M.S., 1992. Intelligence and Changes in Regional Cerebral Glucose Metabolic Rate Following Learning. Intelligence 16, 415-426.

Heinzel, S., Lorenz, R.C., Brockhaus, W.-R., Wustenberg, T., Kathmann, N., Heinz, A., Rapp, M.A., 2014. Working Memory Load-Dependent Brain Response Predicts Behavioral Training Gains in Older Adults. J. Neurosci. 34, 1224-1233. doi:10.1523/JNEUROSCI.2463-13.2014 Hohwy, J., Roepstorff, A., Friston, K., 2008. Predictive coding explains 
1040

1041

1042

1043

1044

1045

1046

1047

1048

1049

1050

1051

1052

1053

1054

1055

1056

1057

1058

1059

1060

1061

1062

1063

1064

binocular rivalry: an epistemological review. Cognition 108, 687-701. doi:10.1016/j.cognition.2008.05.010

Johansen-Berg, H., Baptista, C.S., Thomas, A.G., 2012. Human structural plasticity at record speed. Neuron 73, 1058-60.

doi:10.1016/j.neuron.2012.03.001

Kanwisher, N., McDermott, J., Chun, M.M., 1997. The fusiform face area: a module in human extrastriate cortex specialized for face perception. J. Neurosci. 17, 4302-11.

Koush, Y., Zvyagintsev, M., Dyck, M., Mathiak, K. a, Mathiak, K., 2012. Signal quality and Bayesian signal processing in neurofeedback based on realtime fMRI. Neuroimage 59, 478-89. doi:10.1016/j.neuroimage.2011.07.076

Lange, F.P. De, Heilbron, M., Kok, P., 2018. How Do Expectations Shape Perception ? Trends Cogn. Sci. 22, 764-779. doi:10.1016/j.tics.2018.06.002

Levelt, W.J.M., 1966. The alternation process in binocular rivalry. Br. J. Psychol. 57, 225-238.

Li, W., He, H., Lu, J., Lv, B., Li, M., Jin, Z., 2009. <title>Detection of wholebrain abnormalities in temporal lobe epilepsy using tensor-based morphometry with DARTEL</title> 7497, 749723-749723-6. doi:10.1117/12.833128

Li, X., Hartwell, K.J., Borckardt, J., Prisciandaro, J.J., Saladin, M.E., Morgan, P.S., Johnson, K.A., LeMatty, T., Brady, K.T., George, M.S., 2013. Volitional reduction of anterior cingulate cortex activity produces decreased cue craving in smoking cessation: a preliminary real-time fMRI 

study. Addict. Biol. 18, 739-748. doi:10.1111/j.1369-1600.2012.00449.x

1066

1067

1068

1069

1070

1071

1072

1073

1074

1075

1076

1077

1078

1079

1080

1081

1082

1083

1084

1085

1086

1087

1088

1089

McCarthy, G., Puce, A., Gore, J.C., Allison, T., 1997. Face-Specific Processing in the Human Fusiform Gyrus. J. Cogn. Neurosci. 9, 605610. doi:10.1162/jocn.1997.9.5.605

Miranda, R.A., Casebeer, W.D., Hein, A.M., Judy, J.W., Krotkov, E.P., Laabs, T.L., Manzo, J.E., Pankratz, K.G., Pratt, G.A., Sanchez, J.C., Weber, D.J., Wheeler, T.L., Ling, G.S.F., 2015. DARPA-funded efforts in the development of novel brain - computer interface technologies. J. Neurosci. Methods 244, 52-67.

O'Craven, K.M., Kanwisher, N., 2000. Mental imagery of faces and places activates corresponding stiimulus-specific brain regions. J. Cogn. Neurosci. 12, 1013-23.

Orban, G. a, Zhu, Q., Vanduffel, W., 2014. The transition in the ventral stream from feature to real-world entity representations. Front. Psychol. 5, 695. doi:10.3389/fpsyg.2014.00695

Parker, A., Alais, D., 2007. A bias for looming stimuli to predominate in binocular rivalry. Vision Res. 47, 2661-74. doi:10.1016/j.visres.2007.06.019

Poldrack, R.A., 2015. Is "efficiency" a useful concept in cognitive neuroscience? Dev. Cogn. Neurosci. 11, 12-17. doi:10.1016/j.den.2014.06.001

Rademaker, R.L., Pearson, J., 2012. Training Visual Imagery: Improvements of Metacognition, but not Imagery Strength. Front. Psychol. 3, 224. doi:10.3389/fpsyg.2012.00224

Sagi, Y., Tavor, I., Hofstetter, S., Tzur-Moryosef, S., Blumenfeld-Katzir, T., 
1090

1091

1092

1093

1094

1095

1096

1097

1098

1099

1100

1101

1102

1103

1104

1105

1106

1107

1108

1109

1110

1111

1112

1113

1114 Thibault, R.T., MacPherson, A., Lifshitz, M., Roth, R.R., Raz, A., 2018.

Shibata, K., Watanabe, T., Sasaki, Y., Kawato, M., 2011. Perceptual learning incepted by decoded fMRI neurofeedback without stimulus presentation. Science 334, 1413-5. doi:10.1126/science.1212003

Sitaram, R., Ros, T., Stoeckel, L., Haller, S., Scharnowski, F., Lewis-Peacock, J., Weiskopf, N., Blefari, M.L., Rana, M., Oblak, E., Birbaumer, N., Sulzer, J., 2016. Closed-loop brain training: the science of neurofeedback. Nat. Rev. Neurosci. doi:10.1038/nrn.2016.164

Subramanian, L., Hindle, J. V, Johnston, S., Roberts, M. V, Husain, M., Goebel, R., Linden, D., 2011. Real-time functional magnetic resonance imaging neurofeedback for treatment of Parkinson's disease. J. Neurosci. 31, 16309-17. doi:10.1523/JNEUROSCI.3498-11.2011

Summerfield, C., Koechlin, E., 2008. A neural representation of prior information during perceptual inference. Neuron 59, 336-47. doi:10.1016/j.neuron.2008.05.021

Tartaglia, E.M., Bamert, L., Mast, F.W., Herzog, M.H., 2009. Human perceptual learning by mental imagery. Curr. Biol. 19, 2081-5. doi:10.1016/j.cub.2009.10.060

Taschereau-Dumouchel, V., Cortese, A., Chiba, T., Knotts, J.D., Kawato, M., Lau, H., 2018. Towards an unconscious neural reinforcement intervention for common fears. Proc. Natl. Acad. Sci. 201721572. doi:10.1073/pnas.1721572115 
1115

1116

1117

1118

1119

1120

1121

1122

1123

1124

1125

1126

1127

1128

1129

1130

1131

1132

1133

1134

1135

1136

1137

1138

1139

Neurofeedback with fMRI: A critical systematic review. Neuroimage 172, 786-807. doi:10.1016/j.neuroimage.2017.12.071

Tong, F., Meng, M., Blake, R., 2006. Neural bases of binocular rivalry. Trends Cogn. Sci. 10, 502-511. doi:10.1016/j.tics.2006.09.003

Tong, F., Nakayama, K., Vaughan, J.T., Kanwisher, N., 1998. Binocular rivalry and visual awareness in human extrastriate cortex. Neuron 21, 753-9.

Vartanian, O., Jobidon, M.-E., Bouak, F., Nakashima, A., Smith, I., Lam, Q., Cheung, B., 2013. Working memory training is associated with lower prefrontal cortex activation in a divergent thinking task. Neuroscience 236, 186-194. doi:10.1016/j.neuroscience.2012.12.060

Wang, Y., Yuan, L., Shi, J., Greve, A., Ye, J., Toga, A.W., Reiss, A.L., Thompson, P.M., 2013. Applying tensor-based morphometry to parametric surfaces can improve MRI-based disease diagnosis. Neuroimage 74, 209-30. doi:10.1016/j.neuroimage.2013.02.011

Watanabe, T., Náñez, J.E., Koyama, S., Mukai, I., Liederman, J., Sasaki, Y., 2002. Greater plasticity in lower-level than higher-level visual motion processing in a passive perceptual learning task. Nat. Neurosci. 5, 10031009. doi:10.1038/nn915

Watanabe, T., Náñez, J.E., Sasaki, Y., 2001. Perceptual learning without perception. Nature 413, 844-848. doi:10.1038/35101601

Watanabe, T., Sasaki, Y., Shibata, K., Kawato, M., 2017. Advances in fMRI Real-Time Neurofeedback. Trends Cogn. Sci. 21, 997-1010. doi:10.1016/j.tics.2017.09.010

Weiskopf, N., Mathiak, K., Bock, S.W., Scharnowski, F., Veit, R., Grodd, W., Goebel, R., Birbaumer, N., 2004. Principles of a brain-computer interface 

$(\mathrm{BCl})$ based on real-time functional magnetic resonance imaging (fMRI).

1141 IEEE Trans. Biomed. Eng. 51, 966-70. doi:10.1109/TBME.2004.827063

1142 Welch, K. a, Moorhead, T.W., Mclntosh, a M., Owens, D.G.C., Johnstone,

1143 E.C., Lawrie, S.M., 2013. Tensor-based morphometry of cannabis use on

1144 brain structure in individuals at elevated genetic risk of schizophrenia.

1145 Psychol. Med. 43, 2087-96. doi:10.1017/S0033291712002668

1146

1147

1148 


\section{Acknowledgements}

1150 JE's contribution was supported by a Wellcome Trust Clinical Research 1151 Fellowship.

1152 G.R.R and A.R were supported by The Wellcome Trust Centre for

1153 Neuroimaging core funding from the Wellcome Trust 091593/Z/10/Z.

1154 EF was supported by core funding of the School of Psychology and Language 1155 Sciences, University of Reading.

1156 FS and Y.K were supported by a grant from the Swiss National Science 1157 Foundation (BSSG10_155915)

1158 NW's contribution was supported by the BRAINTRAIN European research 1159 network (Collaborative Project) supported by the European Commission 1160 under the Health Cooperation Work Programme of the 7th Framework 1161 Programme (Grant agreement $n^{\circ}$ 602186). The Wellcome Trust Centre for 1162 Neuroimaging is supported by core funding from the Wellcome Trust 1163 0915/Z/10/Z. The Wellcome Trust Centre for Neuroimaging has an 1164 institutional research agreement with and receives support from Siemens 1165 Healthcare.

1166 GR's contribution was supported by a Wellcome Trust Senior Research 1167 Fellowship.

\section{Author contributions}

1169 J.E, F.S, and G.R designed the experiment. J.E, J.S.W, G.R.R, F.S and G.R 1170 discussed and planned data analysis. J.E collected and analysed the data. 
1171 J.E, Y.K, G.R.R and F.S. developed the real-time acquisition and analysis 1172 tools. J.E and E.F performed the mental imagery control experiment. A.R 1173 performed the CVA analysis. J.E and G.R wrote the paper. All authors read 1174 and commented on the manuscript.

1175 


\section{Figure legends}

1177 Figure 1. Experiment procedure schematic.

1178 Stage 1 Pre-training $B R$

1179 Stage 2 Neurofeedback training: 10 participants were separated into two 1180 groups, a 'face' group and a 'house group', and were trained to increase a 1181 fluctuating thermometer bar (blue bar), up to a fixed mark (orange bar). After 1182 the neurofeedback training sessions, the participants performed a transfer 1183 session with brain modulation in the absence of neurofeedback signal.

1184 Stage 3 Post-training BR: Three types of sessions: a) BR; b) BR with 1185 'concurrent trained upregulation'; and c) BR with 'concurrent non-trained 1186 mental imagery'. 
1188 Figure 2A. Schematic showing group ROIs (FFA and PPA statistical masks) on 1189 inflated canonical brains. Activation was extracted from these regions for 1190 production of the differential signal for neurofeedback training. The direction 1191 of regulation of these ROIs was specific for each group i.e. House Group, PPA 1192 up/ FFA down, Face Group, FFA up/ PPA down.

1194 Figure 2B. Mean BOLD signal changes across groups, in the strategy-related 1195 ROI (red) and the strategy-unrelated ROI (blue), for each of the nine training 1196 sessions. The green line shows the difference in mean BOLD activation 1197 between the two brain regions and corresponds to the neurofeedback training 1198 signal that participants visualised in the scanner as a fluctuating bar. Error 1199 bars show \pm 1 SEM. 
1202 Figure 3A. Cumulative dominance durations across participants for pre1203 training BR, and the three post-training BR sessions: Post-training, Post1204 training BR with concurrent trained upregulation, and Post-training BR with 1205 concurrent non-trained mental imagery. Error bars show \pm 1 SEM. The total 1206 duration of each BR block was 40s.

1207 
1208 Figure 3B. Changes in cumulative dominance durations for binocular rivalry 1209 (BR) sessions, showing comparisons before and after neurofeedback training 1210 collapsed across both groups. Error bars indicate \pm 1 SEM

1211

1212 A. Pre/post training BR comparison

1213 B. Pre/post-training BR with concurrent training upregulation

1214 C. Pre/post-training BR with concurrent non-trained mental imagery

1215

$1216{ }^{*} p<0.05$. Double ${ }^{* *} p<0.01$. Horizontal brackets indicate significant differences in 1217 the changes of cumulative dominance durations $(p<0.05) \sim$ over a bracket 1218 indicates $\mathrm{p}=\mathbf{0 . 0 7}$.

1219

1220 
1221 Figure 4. BOLD activation changes in the trained ROls, during binocular rivalry 1222 (BR) sessions, before and after neurofeedback training. There was a significant 1223 reduction in activation in both the strategy-related ROI and the strategy1224 unrelated ROI following training. When BR was performed with concurrent 1225 trained up-regulation, there was a significant further decrease in BOLD 1226 activation in the strategy-unrelated ROI only. Error bars indicate \pm 1 SEM. (* $1227 \quad p<0.05)$.

1228 
1. Pre-training BR

2. Neurofeedback training 2 groups of 5 participants:

-FFA minus PPA signal 'face' strategies

-PPA minus FFA signal 'house' strategies

\section{Post-training BR}

a)

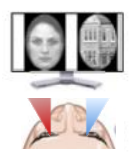

BR

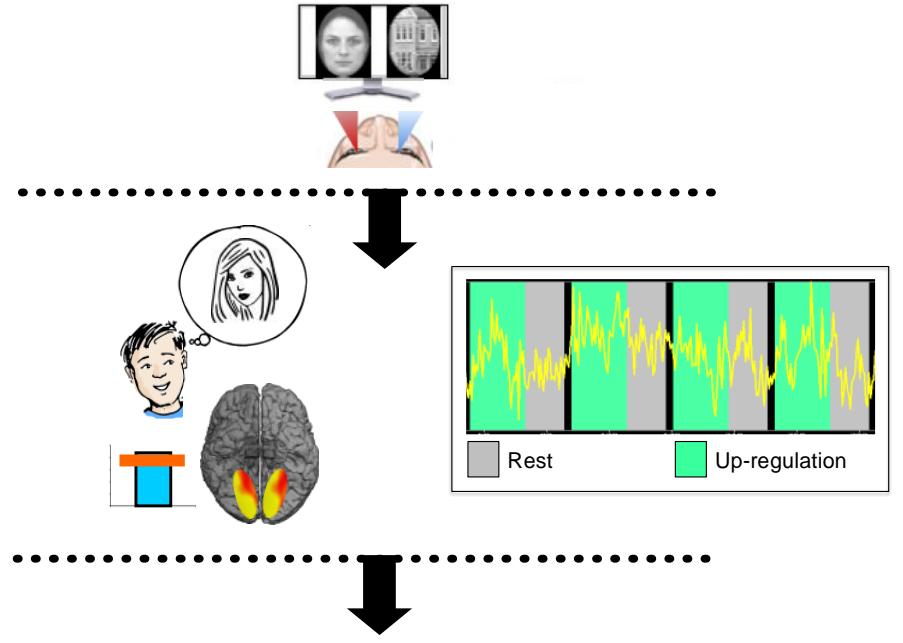

b)

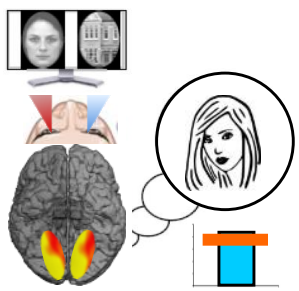

$\mathrm{BR}+$ Trained Upregulation c)

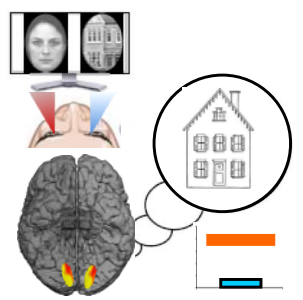

$\mathrm{BR}+$ Non-trained Mental imagery

Figure 1 

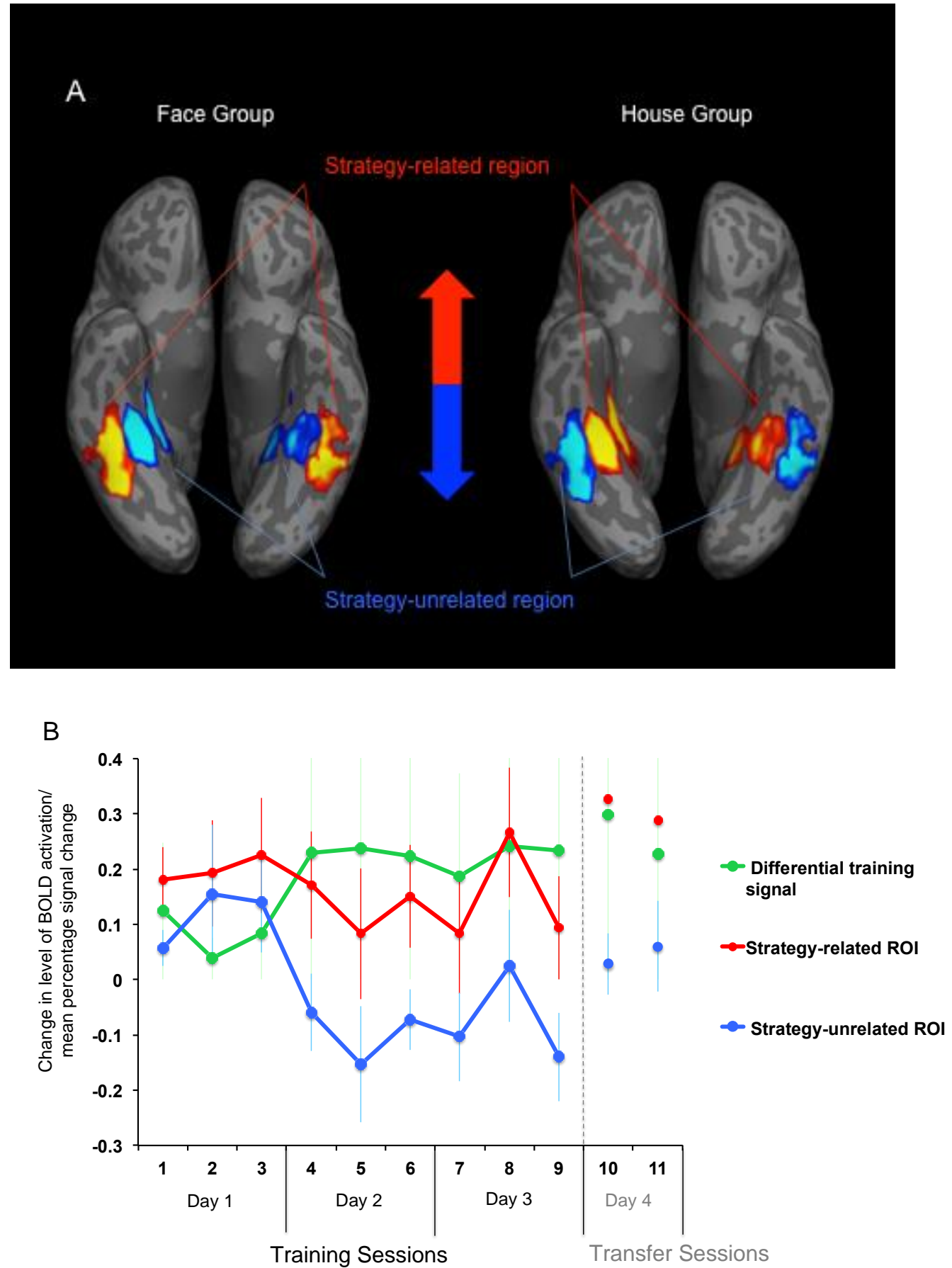

1236 Figure 2A and B. 


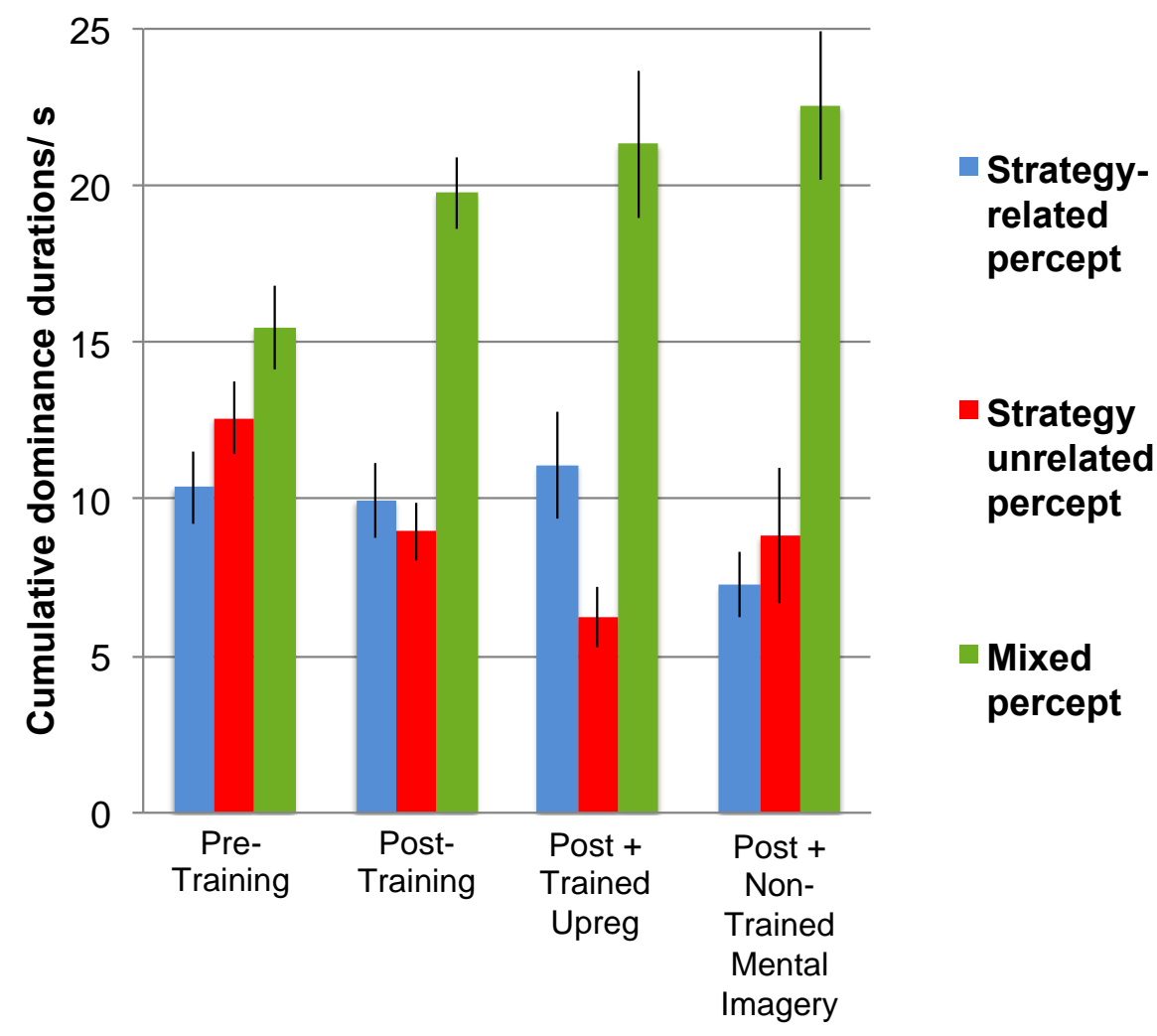

Figure 3A. 


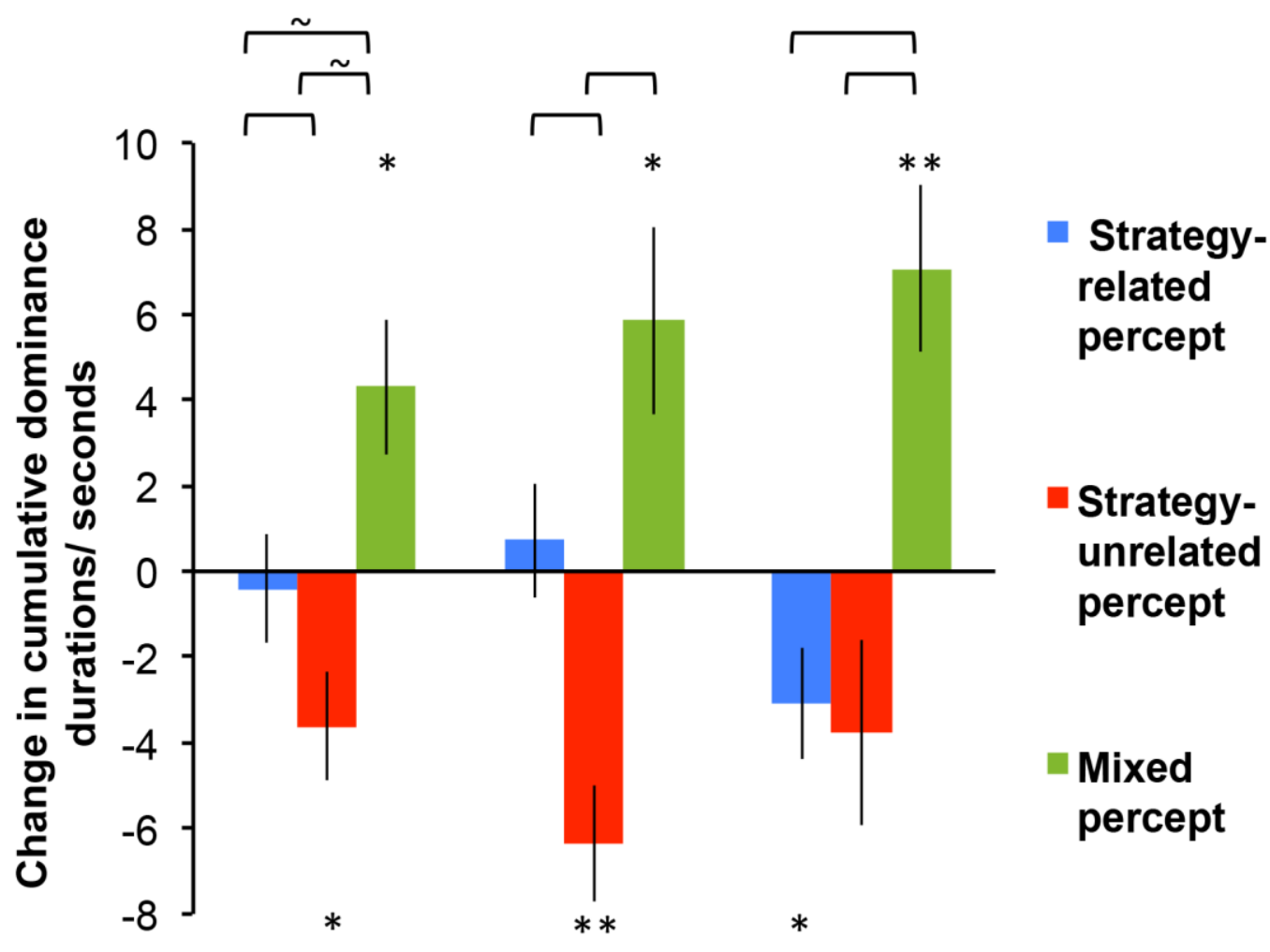
A. Pre vs
Post
B. Pre vs
Post
C. Pre vs
+Trained
Post
upregulation
+ Non-trained
mental Imagery

Binocular Rivalry Sessions Comparison

Figure 3B.

1244 


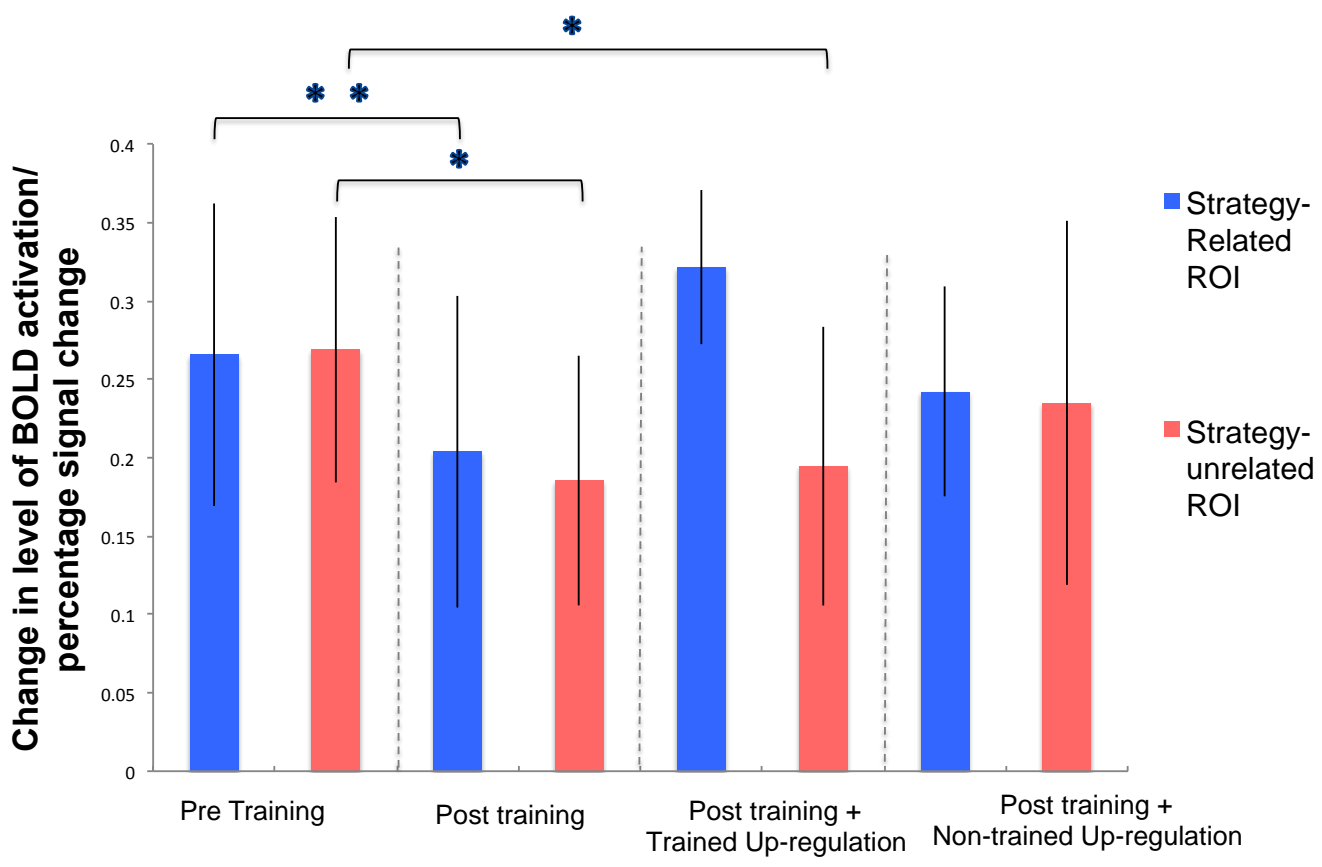

Figure 4. 


\section{Supplementary Materials List:}

1250

- Supplementary methods

1251 - Supplementary results

1252 - Supplementary discussion

1253 - Supplementary references

1254 - Supplementary figures S1-S4

1255

1256

1257 Supplementary Methods

1258

1259 Learning Effect across Rt-fMRI Neurofeedback Training

1260

1261 The learning effect measures the change in BOLD activation in trained brain

1262 region/s across the neurofeedback training sessions. The mean percentage

1263 signal change (PSC) for each training run and ROI was calculated and plotted

1264 (seeFigure2B in main paper, and compare with S1, S2).

1265

1266

1267

1268 


\section{Supplementary Results}

\section{Results - Behaviour}

1271

1272

\section{Binocular Rivalry - Durations:}

1273 See main paper.

1274

1275 Results - Imaging

1276

1277 Strategy-related and Strategy-unrelated ROls:

1278 We first determined if the differential signal significantly changed over days 1279 across participants (see main paper, Result Section). We additionally 1280 examined the changes in the two ROls used to produce the differential signal; 1281 the strategy-related ROI and the strategy-unrelated ROI (see Figure 2, main 1282 paper).

1283

1284 A one-way ANOVA (with 3 levels corresponding to the 3 training days) 1285 revealed a significant reduction in activation in the strategy-unrelated $\mathrm{ROI}$ 1286 over the 3 days of training $(F(2,16)=8.71, p=0.003)$. On the other hand, a 1287 one-way ANOVA for the strategy-related ROI revealed no significant change $1288(F(2,16)=0.33, p=0.72)$.

1289

1290 Sub-groups:

1291 To assess whether there was any difference between the face and house 1292 group during training, an ANOVA was performed on the differential training 1293 signal across the 3 training days, with a between-subjects factor with two 
1294 levels (for the two sub-groups, 'Face' and 'House'). This did not reveal a 1295 significant interaction $(F(2,14)=0.064, p=0.94)$ between the two factors.

1296

1297 For neurofeedback training graphs for the two groups (mean percentage 1298 signal change over 9 sessions), please see Figures S2 and S3.

1299

1300

1301 


\section{Supplementary Discussion}

1303

\section{Levelt's Second Proposition, 1966}

1305 Levelt's second proposition(Levelt, 1966), as applied to stimulus perception 1306 was based on the physical properties of visual stimuli and states: "Variation of 1307 the stimulus strength in one eye will only influence the mean dominance 1308 duration of the contralateral eye and not the mean dominance duration of the 1309 ipsilateral eye”.

\section{Known Influences on Visual Perception}

1312 The role of 'priming' and 'cueing' might also be invoked as possible causes for 1313 the perceptual changes observed following neurofeedback training in this 1314 study. Prior presentation of a specific orientation grating can cause an 1315 increase in the perception of the identical grating during BR. However, 1316 dominance durations were unchanged(Denison et al., 2011). Similarly, 1317 exogenous cueing prior to BR can increase the probability of the predominant 1318 percept being linked to the cue. For example prior to BR, hearing sentences 1319 with the word 'face', results in FFA activation(Pelekanos et al., 2011). 1320 Nonetheless, no significant change in stimulus dominance between faces and 1321 houses on rivalry trials were observed when participants were cued with a 1322 word linked to one of the rivalrous stimuli. Dominance durations have also 1323 been demonstrated as being immune to the effects of volitional attention(Jung 1324 et al., 2016), and reflective of true differences in sensory processing(Dieter et 1325 al., 2016). It is therefore unlikely that the perceptual changes produced by 1326 neurofeedback training could be ascribed to participant expectation. Evidently, 
neither altering the level of activity in higher order brain regions involved in perception, nor applying known influences on visual perception, provide a comprehensive explanation for the lasting shifts in perceptual bistability observed following neurofeedback training in this study.

\section{Controlling the Neurofeedback Signal}

1333 With regards to the neurofeedback training signal itself (i.e. differential brain 1334 activation between two ROIs), there were five potential activation states which 1335 could increase the difference between the two brain regions (strategy-related 1336 ROI minus strategy-unrelated $R O I$ ), leading to upregulation of the training 1337 signal: These could be: (1) an increase in strategy-related ROl; (2) a decrease 1338 in strategy-unrelated ROI; (3) a combination of the two; (4) a relatively greater 1339 increase in strategy-related ROI as compared to strategy-unrelated ROI; and 1340 (5) a relatively greater decrease in the strategy-unrelated ROI. Based on our 1341 results (Figure $1 \mathrm{~B}$ in main paper), the mechanism for the upregulation of the 1342 differential signal across groups during neurofeedback training appeared to be 1343 produced by maintenance of activation in the strategy-related ROI, and a 1344 reduction of activation in the strategy-unrelated $\mathrm{ROI}$. 


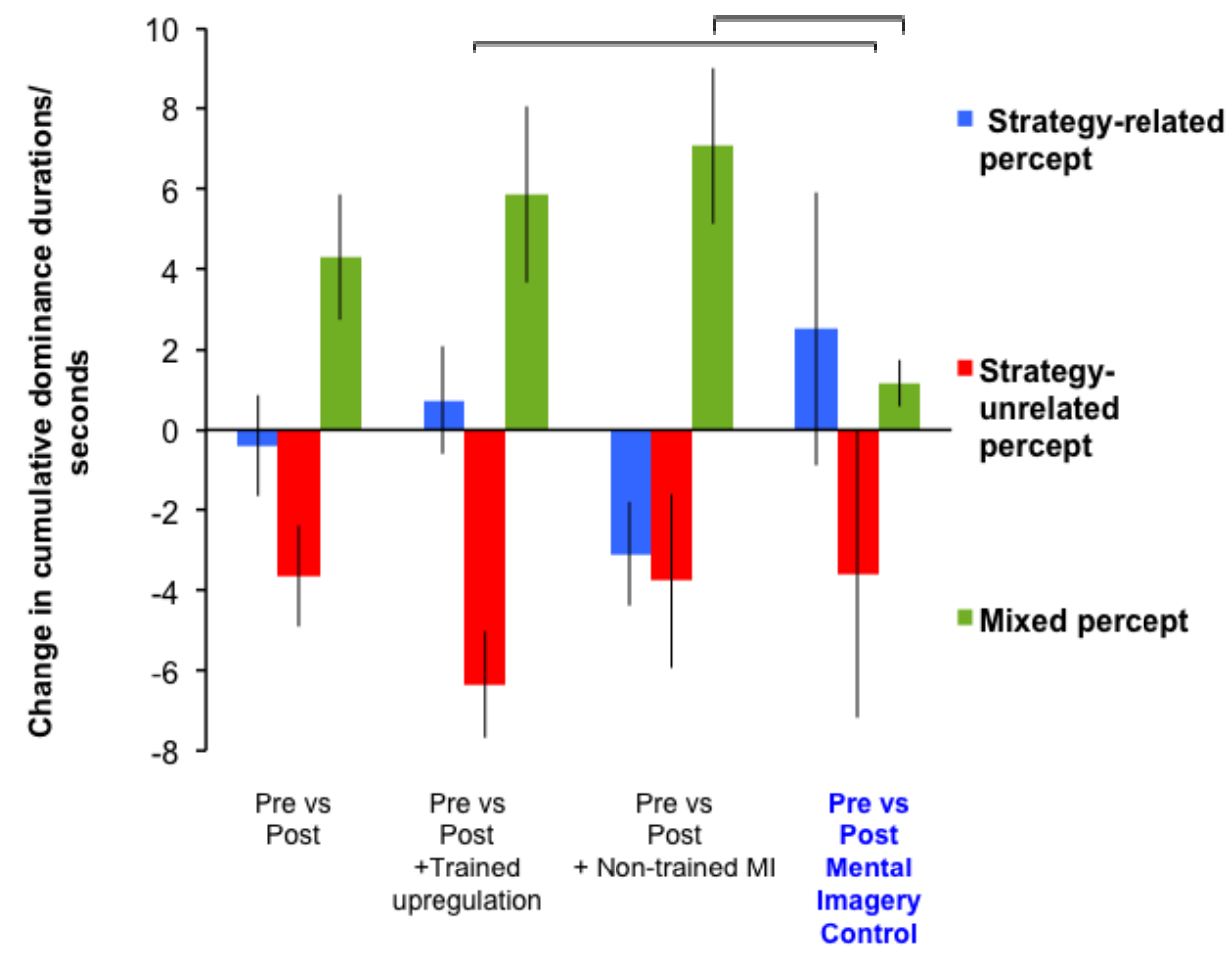

1352 Figure S1. Changes in cumulative dominance durations for binocular rivalry 1353 sessions, showing comparisons before and after neurofeedback training. This 1354 figure is the analogous to Figure 3B in the main paper, but additionally shows 1355 changes in dominance durations for the 'Mental Imagery' control group. Error 1356 bars indicate \pm 1 SEM. Horizontal brackets show significant between group 1357 comparisons for percepts $(p<0.05)$.

B. Pre vs. Post-training BR with concurrent training up-regulation
D. Pre vs. Post training BR comparison for Mental Imagery Control group 


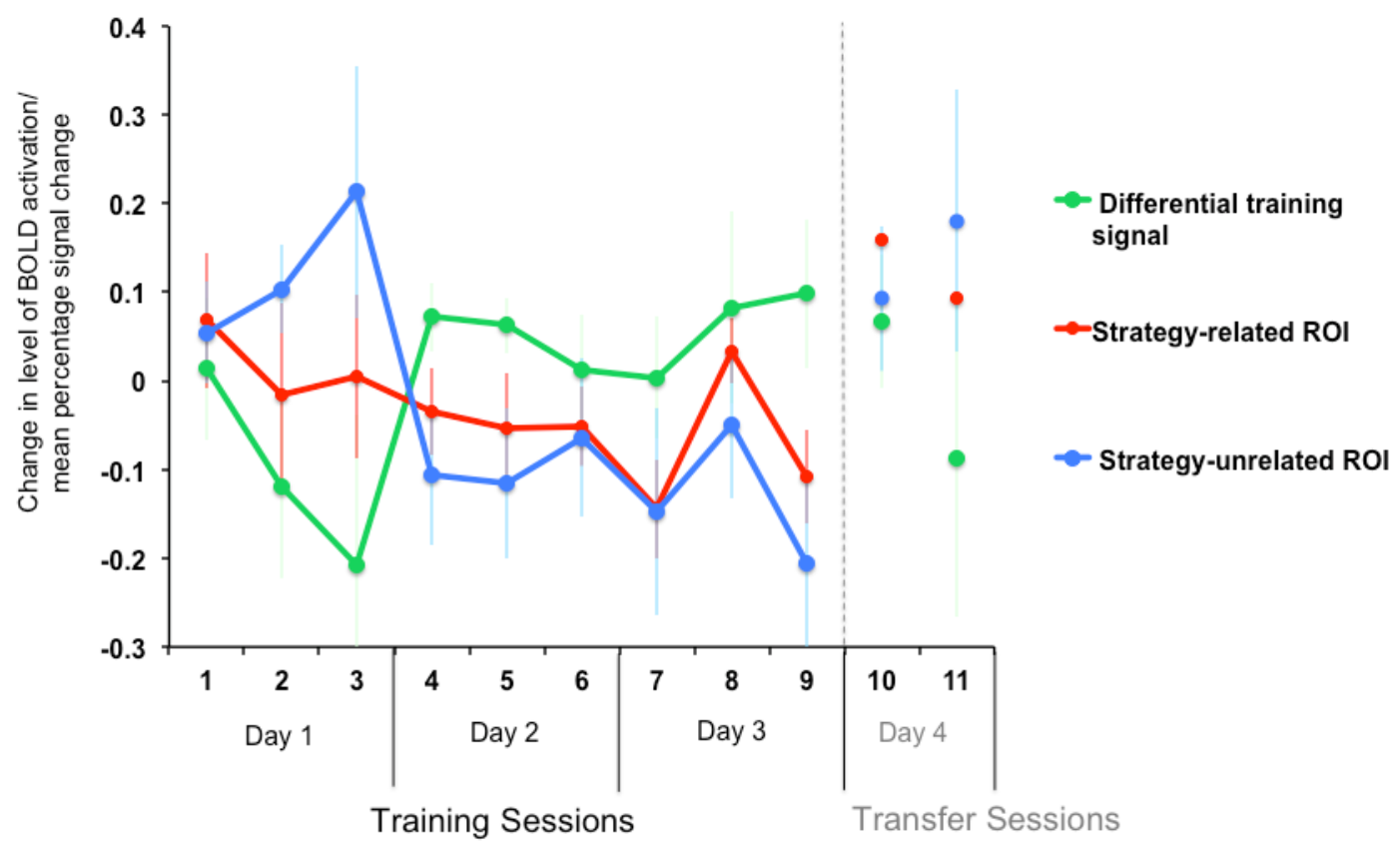

1366 Figure S2. Mean BOLD signal changes across the House group, in the strategy1367 related brain region (red) and the strategy-unrelated brain region (blue), for 1368 each of the nine training sessions. The green line shows the difference in mean 1369 BOLD activation between the two brain regions and corresponds to the 1370 neurofeedback training signal. Error bars show \pm 1 SEM. 


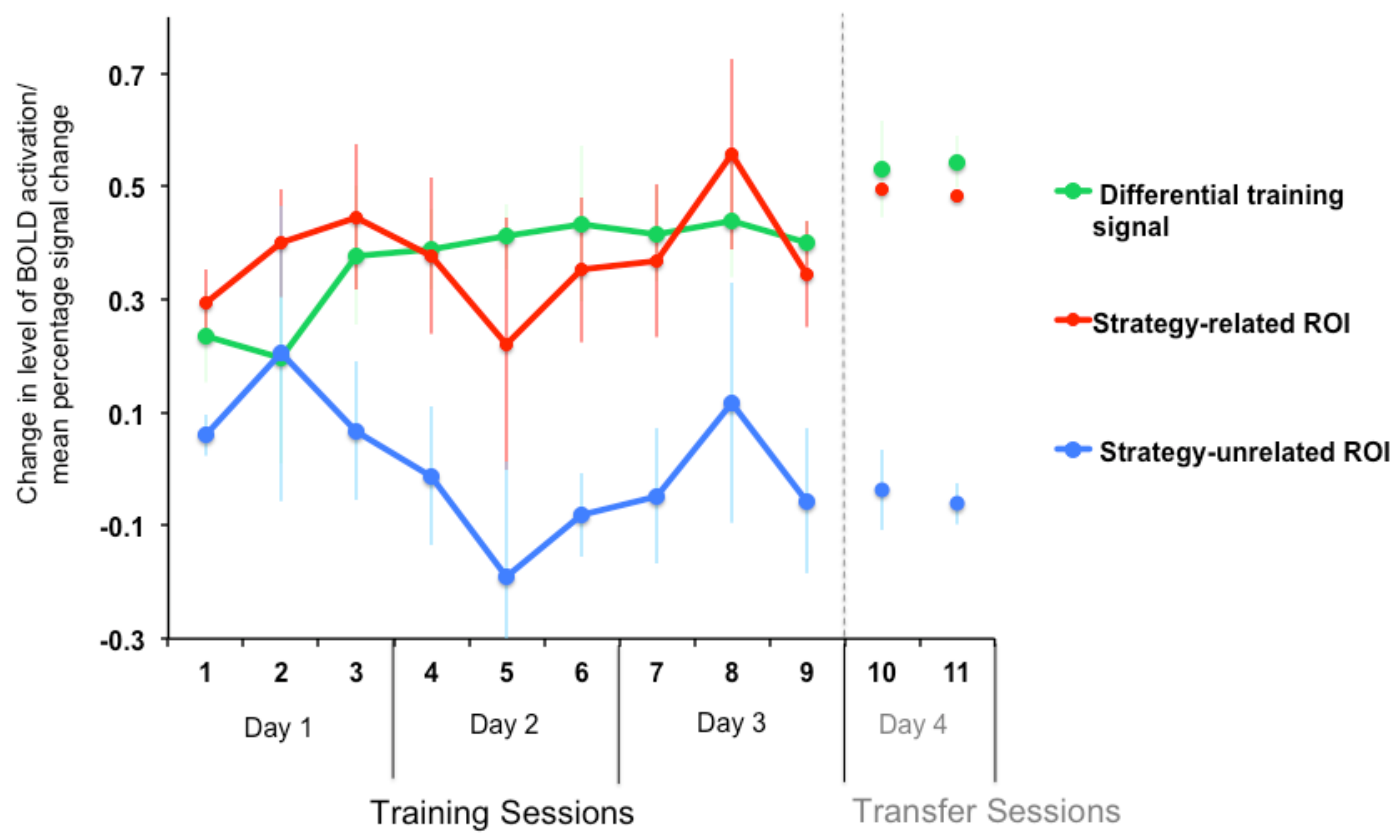

1377 Figure S3. Mean BOLD signal changes across the Face group, in the strategy1378 related brain region (red) and the strategy-unrelated brain region (blue), for 1379 each of the nine training sessions. The green line shows the difference in mean 1380 BOLD activation between the two brain regions and corresponds to the 1381 neurofeedback training signal. Error bars show \pm 1 SEM. 
A
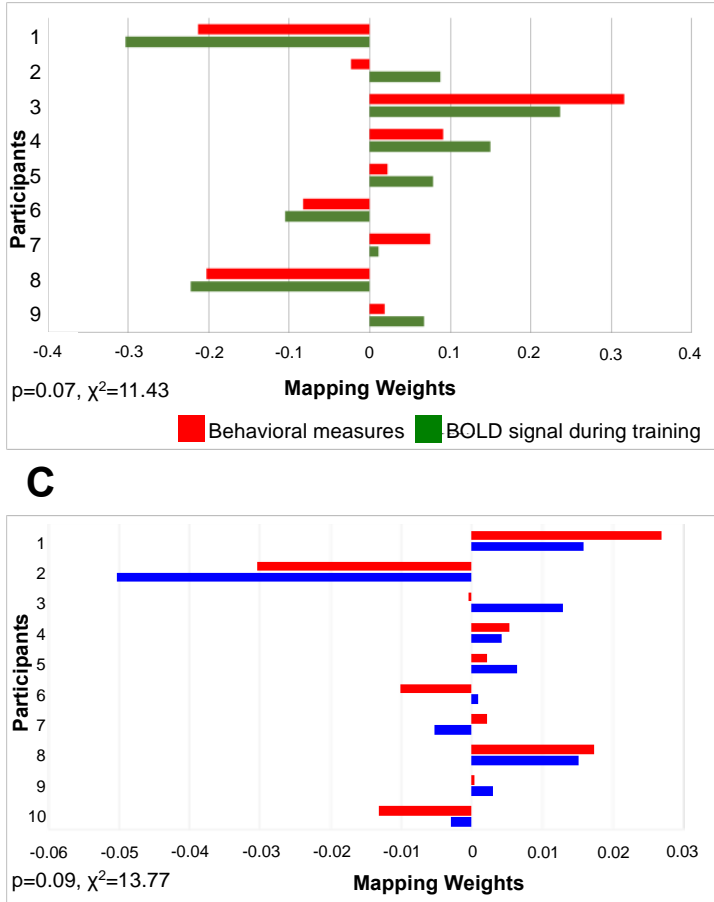

1387 variables following neurofeedback training.
B

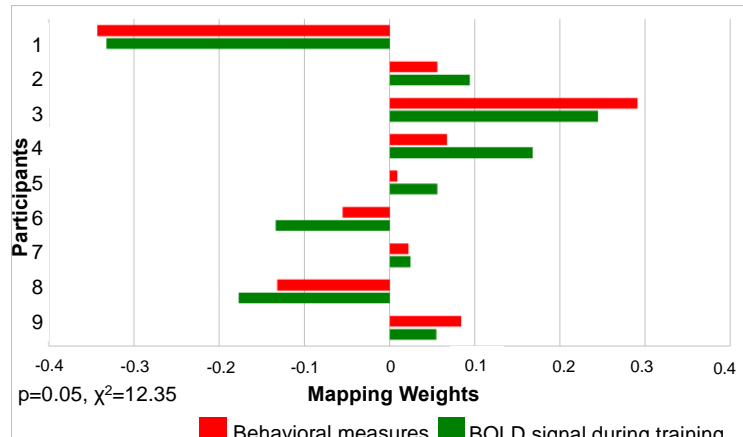

D

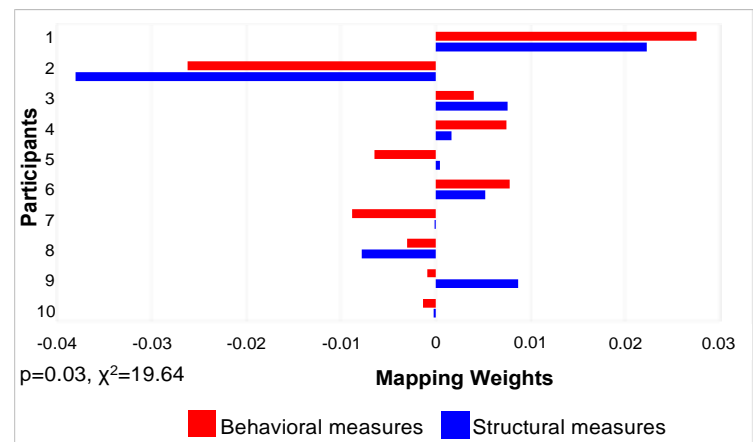

Figure S4. Canonical variate analysis illustrating the correlation between individual behaviour and physiological measures. For each participant mapping weights are shown for pairs of predictor and outcome variables. This approach aims to reveal relationships that may exist between multiple outcome

A,B: Comparison of BR behavioural measures (i.e. durations of mixed, strategy-related and strategy-unrelated percepts), and functional BOLD signal changes across training (i.e. differential signal). Nine of the ten participants were included, as one of the participants did not complete all nine training sessions. Participants 1-5 are Face Group, Participants 6-9 are House Group. A shows a non-significant relationship $(p=0.07)$ between individual participant BR measures (pre vs. post training) and functional BOLD signal changes across training. B shows a non-significant relationship $(p=0.05)$ between 
1403 individual participant BR measures (pre vs. post-training with concurrent 1404 trained upregulation) and functional BOLD signal changes across training.

1405

1406 C,D: Comparison of BR behaviour measures (i.e. durations of mixed, strategy1407 related and strategy-unrelated percepts), and structural measures from FFA 1408 and PPA (pre vs. post training). Participants 1-5 are 'Face Group’, Participants 1409 6-10 are 'House Group'. C shows a non-significant relationship ( $p=0.09)$ 1410 between individual participant BR measures (pre vs. post training) and 1411 structural measures from FFA, and PPA (pre vs. post training). D shows a 1412 significant relationship $(p=0.03)$ between individual participant BR measures 1413 (pre vs. post-training with concurrent trained upregulation) and structural 1414 measures from FFAand PPA (pre vs. post training). 


\section{Supplementary References}

1417 Denison, R.N., Piazza, E. a, Silver, M. a, 2011. Predictive Context Influences 1418 Perceptual Selection during Binocular Rivalry. Front. Hum. Neurosci. 5, 1419 166. doi:10.3389/fnhum.2011.00166

1420 Dieter, K.C., Sy, J.L., Blake, R., 2016. Individual differences in sensory eye 1421 dominance reflected in the dynamics of binocular rivalry. Vision Res. 1422 doi:10.1016/j.visres.2016.09.014

1423 Jung, Y., Kang, M.-S., Chong, S.C., 2016. Effect of Attention on the Initiation 1424 of Binocular Rivalry. Perception 45, 492-504.

1425 doi:10.1177/0301006615622324

1426 Levelt, W.J.M., 1966. The alternation process in binocular rivalry. Br. J.

$1427 \quad$ Psychol. 57, 225-238.

1428 Pelekanos, V., Roumani, D., Moutoussis, K., 2011. The effects of categorical 1429 and linguistic adaptation on binocular rivalry initial dominance. Front. $1430 \quad$ Hum. Neurosci. 5, 187. doi:10.3389/fnhum.2011.00187 1431 1432 1433 1434 